\title{
On the Stability and Accuracy of the BGK, MRT and RLB Boltzmann Schemes for the Simulation of Turbulent Flows
}

\author{
Patrick Nathen ${ }^{1, *}$, Daniel Gaudlitz ${ }^{2}$, Mathias J. Krause ${ }^{3}$ and \\ Nikolaus A. Adams ${ }^{1}$ \\ ${ }^{1}$ Insitute of Aerodynamics and Fluid Mechanics, Technische Universität München, \\ Boltzmannstrasse 15, 85748 Garching bei München, Germany. \\ 2 Institute of Automotive Engineering, University of Applied Sciences Zwickau, \\ Scheffelstraße 39, 08066 Zwickau, Germany. \\ ${ }^{3}$ Institute for Mechanical Process Engineering and Mechanics, Institute for Applied \\ and Numerical Mathematics, Karlsruhe Institute of Technology, Strasse am Forum 8, \\ 76131 Karlsruhe, Germany.
}

\begin{abstract}
This paper presents an analysis of the stability and accuracy of different Lattice Boltzmann schemes when employed for direct numerical simulations of turbulent flows. The Single-Relaxation-Time scheme of Bhatnagar, Gross and Krook (BGK), the Multi-Relaxation-Time scheme (MRT) and the Regularized Lattice Boltzmann scheme (RLB) are considered. The stability and accuracy properties of these schemes are investigated by computing three-dimensional Taylor-Green vortices representing homogeneous isotropic turbulent flows. Varying Reynolds numbers and grid resolutions were considered. As expected, the BGK scheme requires sufficiently high grid resolutions for stable and accurate simulations. Surprisingly, the MRT scheme when used without any turbulence model fails to obtain mesh convergence for the type of flow considered here. The RLB scheme allows for stable simulations but exhibits a strong dissipative behavior. A similar behavior was found when employing the mentioned LBM schemes for numerical simulations of turbulent channel flows at varying Reynolds numbers and resolutions. The obtained insights on accuracy and stability of the considered Lattice Boltzmann methods can become useful especially for the design of effective turbulence models to be used for high Reynolds number flows.
\end{abstract}

Key words: Lattice Boltzmann, homogeneous isotropic turbulence, wall bounded turbulent flows.

\footnotetext{
*Corresponding author. Email addresses: patrick. nathen@aer.mw.tum.de (P. Nathen), Daniel.Gaudlitz@aer.mw.tum.de (D. Gaudlitz), mathias.krause@kit.edu (M. J. Krause), nikolaus.adams@tum.de (N. A. Adams)
} 


\section{Introduction}

In the last three decades, the Lattice Boltzmann Method (LBM) became a promising alternative to conventional methods, like solving the Navier-Stokes equations with Finite Volumes or Finite Elements. Flows through porous media, multi-phase and multicomponent flows with and without heat transfer as well as flows around complex geometries have been investigated by several authors, see, e.g. [1,7]. With LBM the computational domain is discretized by an equidistant mesh, on which a discrete set of velocity distribution functions is solved numerically. This set of velocity distribution functions corresponds to discrete lattice velocities, which are used to recover the macroscopic moments in terms of a Hermite Polynomial expansion. Although the LBM has been applied to a wide range of fluid-dynamics applications, turbulence modeling in the LBM framework still requires considerable research and has not reached the level of maturity as seen for Navier-Stokes-based methods, see references [37,38] and [39]. Important recent advances have been made by Sagaut et al., see Sagaut [39] and Malaspinas and Sagaut [31,32]. The approach proposed by Malaspinas and Sagaut [31] is based on the Approximate Deconvolution Method (ADM) of Stolz and Adams [40]. The discrete Boltzmann equations are filtered and subsequently deconvoluted with a regularized inverse filter operation in order to reconstruct the proper macroscopic equations for LES within the kinetic theory. The ADM as implemented in [31] is based on the BGK collision approach, yet it can be extended for any other standard collision operator like the MRT and the RLB approach. Due to the substantial differences of the different collision models, see also Section 2, it is crucial to understand their properties in terms of stability and accuracy, when used for the simulation of turbulent flows. Moreover, these properties have to be taken into account when adapting turbulence models to LBM schemes.

To the author's knowledge, a comparison of the stability and accuracy of different discrete LBM schemes without turbulence models when applied to three-dimensional turbulent flows of varying Reynolds number and employing different mesh resolutions has not been presented so far. For Navier-Stokes equation based methods it is common practice to investigate the properties of the discretization scheme in terms of spatial and temporal properties. The Lattice Boltzmann algorithm corresponds to a finite difference scheme with spatial accuracy $\mathcal{O}\left(\Delta x^{2}\right)$ and a temporal accuracy $\mathcal{O}(\Delta t)$. This work aims to investigate the stability and accuracy of different discrete collision schemes for the LBM by carrying out resolved and under-resolved Direct Numerical Simulations (DNS) of Homogeneous Isotropic Turbulence at varying Reynolds numbers. Since no turbulence models will be employed, the observed dissipation will be only due to viscous effects and numerical dissipation of the selected collision scheme. For selected LBM schemes we will provide insights on the accuracy and stability, which can be useful for the further development of turbulence models in the LES-LBM framework. The effectiveness of turbulence models strongly depends on the properties of the numerical scheme employed to solve the basic flow equations. For the type of flow considered in this work some of the recently presented LBM schemes can provide stable simulations of appropriate accuracy. 
The Karlin-Bösch-Chikatamarla (KBC) model, see Karlin et al. [21], uses an entropic formulation, which increases stability significantly. This model was developed further by Dorschner et al. [9,10]. Geier [14] and Geier et al. [13] introduced another advanced LB model, which showed excellent stability for the simulation of turbulent flows. However, in this work we investigate the stability and accuracy of three widely used Lattice Boltzmann collision operators: the Bhatnagar, Gross and Krook (BGK), the Multi-RelaxationTime (MRT) and the Regularized Lattice Boltzmann scheme (RLB), when computing the Taylor-Green vortex which represents a typical homogeneous isotropic turbulent flow. For assessing details on numerical stability and accuracy for wall-bounded turbulent flows, we employ these three models also for the turbulent channel flow as a benchmark test case.

The paper is structured as follows: In Section 2 the LBM is introduced briefly, and differences between the Single-Relaxation-Time scheme of Bhatnagar, Gross and Krook (BGK), the Multi-Relaxation-Time (MRT) and the regularized Lattice Boltzmann scheme (RLB) are explained. Simulations of HIT without turbulence models at different Reynolds numbers, mesh resolutions and with different discrete lattice schemes are compared in Section 3. The results of the three LBM schemes to the turbulent channel flow at $R e_{\tau}=180$, $R e_{\tau}=395$ and $R e_{\tau}=590$ for varying resolutions are outlined in Section 4 . Based on spectral analyses of the flow field the lattice schemes are analyzed systematically. The interaction between the discrete schemes and mesh resolutions are investigated and the limitations of individual discrete schemes for the simulation of turbulent flows will be discussed. Finally, conclusions are drawn in Section 5.

\section{The Lattice Boltzmann Methods}

With LBM, a set of kinetic equations in terms of discrete velocity distribution functions $f_{\alpha}(t, x)$ are solved numerically at time $t$ and position $x$. The discrete Boltzmann equations can be written as

$$
f_{\alpha}\left(t+\Delta t, x+c_{\alpha} \Delta t\right)=f_{\alpha}(t, x)+\Omega_{\alpha},
$$

where $\Omega_{\alpha}$ is the collision operator, which represents non-linear and viscous effects and $c_{\alpha}$ with $\alpha=0,1, \cdots, q-1$ is a set of discrete lattice velocities. Depending on the particular scheme (BGK, MRT or RLB), the collision term is modeled in different ways, see $[1,3,7,8$, 28,29]. Macroscopic moments are reconstructed with a Gauss-Hermite quadrature based on the Hermite Polynomial expansion of $f_{\alpha}$ on a discrete lattice. The first two moments of the velocity distribution functions are the conserved moments, namely the density $\rho$ and the momentum $\rho \boldsymbol{u}$, which read

$$
\begin{aligned}
& \rho=\sum_{\alpha} f_{\alpha}, \\
& \rho \boldsymbol{u}=\sum_{\alpha} \boldsymbol{c}_{\alpha} f_{\alpha},
\end{aligned}
$$


while the momentum flux is the second-order off-equilibrium moment of the velocity distribution functions

$$
\Pi=\sum_{\alpha} f_{\alpha}^{n e q} \boldsymbol{c}_{\alpha} \boldsymbol{c}_{\alpha}
$$

In order to reconstruct the macroscopic equations of fluid motion, a Chapman Enskog expansion is used, see references [2,27] among others. In Eq. (2.3) $f_{\alpha}^{\text {neq }}=f_{\alpha}-f_{\alpha}^{e q}$ is the nonequilibrium part of the velocity distribution function. The velocity distribution functions are expanded with a simple multi-scale expansion factor, which is directly related to the Knudsen number $\epsilon$. For the discrete distribution functions this procedure can be written as

$$
f_{\alpha}=f_{\alpha}^{(0)}+\epsilon f_{\alpha}^{(1)}+\epsilon^{2} f_{\alpha}^{(2)}+\cdots
$$

and is subsequently inserted into the discrete Lattice Boltzmann equation, which is Taylorexpanded in space and time up to second order. The macroscopic equations are obtained by taking the zeroth- and first-order velocity moments and a subsequent integration over the velocity space.

To close the evolution equation of the particle distribution function (2.1), the collision term needs to be modeled. One well-known approach is the linearization around small perturbations of the thermodynamic equilibrium $f_{\alpha}^{e q}$. This approach is called the Bhatnagar-Gross-Krook (BGK) ansatz, see [15, 16, 18, 41], which represents the collision term as a linear relaxation towards Maxwellian equilibrium

$$
\Omega_{\alpha}:=-\frac{1}{\tau}\left(f_{\alpha}(t, x)-f_{\alpha}^{e q}(t, x)\right) .
$$

The relaxation time $\tau$ is related to the viscosity and $f_{\alpha}^{e q}$ is a low Mach number truncated Maxwell-Boltzmann distribution, which is adjusted in such a way, that Eq. (2.3) is satisfied and mass and momentum are conserved. A widely used formulation for $f_{\alpha}^{e q}$ is given by

$$
f_{\alpha}^{e q}=\rho \omega_{\alpha}\left[1+\frac{c_{\alpha} u}{c_{s}^{2}}+\frac{1}{2 c_{s}^{4}}\left(\boldsymbol{u} \boldsymbol{u}-c_{s}^{2} \delta\right) \boldsymbol{u} u\right] .
$$

The constant weights $\omega_{\alpha}$ are obtained by a Gauss-Hermite quadrature on the lattice, $c_{s}$ is the lattice speed of sound and $\delta$ is the Kronecker delta. Although, the BGK approach has been applied successfully to a wide range of fluid mechanics, see $[17,43]$, it suffers from instabilities at high Reynolds numbers, which originate from the evolution of moments of $f_{\alpha}$, which can not be attributed directly to physical quantities in fluid mechanics. To remedy this shortcoming, a Multi-Relaxation-Time (MRT) scheme was developed by D'Humiéres et al. [8]. The main idea is to transform the collision step into the momentum space and to relax each moment separately in order to reduce instabilities arising from the temporal growth of such unphysical moments. Thus, the single relaxation time $\tau$ from the BGK model is replaced by a relaxation time matrix $S$, which relaxes each moment $m_{\alpha}:=\boldsymbol{M} f_{\alpha}$ independently. The matrix $\boldsymbol{M}$ is a linear transformation matrix and the 
corresponding algorithm for the MRT scheme reads

$$
f_{\alpha}\left(t+\Delta t, x+c_{\alpha} \Delta t\right)=f_{\alpha}(t, x)-M^{-1} \boldsymbol{S}\left(m_{\alpha}(t, x)-m_{\alpha}^{e q}(t, x)\right) .
$$

Our implementation is based on the original formulation of D'Humiéres et al. [8], where the moments are defined as

$$
m_{\alpha}=\left(\rho, e, \epsilon, j_{x}, q_{x}, j_{y}, q_{y}, j_{z}, q_{z}, 3 p_{x x}, 3 \pi_{x x}, p_{w w}, \pi_{w w}, p_{x y}, p_{y z}, p_{x z}, m_{x}, m_{y}, m_{z}\right)
$$

and the diagonal relaxation time matrix is given by

$$
S=\operatorname{diag}\left(0, s_{1}, s_{2}, 0, s_{4}, 0, s_{4}, 0, s_{4}, s_{9}, s_{10}, s_{9}, s_{10}, s_{13}, s_{13}, s_{13}, s_{16}, s_{16}, s_{16}\right),
$$

where $s_{1}=1.19, s_{2}=s_{10}=1.4, s_{4}=1.2$, and $s_{16}=1.98$. The coefficients $s_{9}$ and $s_{13}$ are related to the viscosity $v$ as

$$
v=\frac{1}{3}\left(\frac{1}{s_{9}}-\frac{1}{2}\right)=\frac{1}{3}\left(\frac{1}{s_{13}}-\frac{1}{2}\right) .
$$

The MRT model has been found to increase the stability of the LBM method substantially. Yet, Freitas et al. [11] have shown that inconsistent formulation of boundary conditions for stresses on domain boundaries, where velocities are prescribed, can also cause instabilities of the MRT model for Reynolds numbers larger than $R e \approx 5000$ for threedimensional flows. More recently, Geier et al. [13] showed stability and accuracy problems for the MRT model at high Reynolds number flows.

In another attempt to suppress instabilities, Latt [28] proposed a regularization of the aforementioned BGK algorithm, employing an approximation of the first-order multiscale expansion term

$$
f_{\alpha}^{n e q}=f_{\alpha}-f_{\alpha}^{e q} \approx f_{\alpha}^{(1)}=-\frac{\Delta t}{\omega c_{s}^{2}} \omega_{\alpha} Q_{\alpha}: \nabla \rho \boldsymbol{u}
$$

Here, $Q_{\alpha}$ is the first-order non equilibrium moment $Q_{\alpha}=\sum c_{\alpha} c_{\alpha} f_{\alpha}^{\text {neq }}$. The non-equilibrium distribution function $f_{\alpha}^{n e q}$ is used to approximate the first-order multiscale expansion term in Eq. (2.4). This term is included in the BGK model, such that the regularized BGK algorithm reads

$$
f_{\alpha}\left(t+\Delta t, x+c_{\alpha} \Delta t\right)=f_{\alpha}(t, x)+(1-\omega) f_{\alpha}^{(1)}(t, x) .
$$

This regularization operation is not only necessary within the flow field, but also at the boundaries. The main issue with respect to boundary conditions in the RLB scheme is the proper reconstruction of the unknown distribution functions propagating into the flow domain. Different approaches to model $f_{\alpha}^{(1)}$ at domain boundaries are proposed in $[29,30]$. All simulations conducted in this work are performed with the open source LBM software openLB, see $[20,23,24]$. 


\section{Homogeneous Isotropic Turbulence: The Taylor-Green Vortex}

By computing the evolution of the well-known three-dimensional Taylor-Green vortex [4], properties of the Lattice Boltzmann collision schemes outlined in Section 2, are investigated. The velocity and density field of a three-dimensional cubic domain of length $2 \pi$ has been initialized with

$$
\begin{aligned}
& u^{0}=\frac{2}{\sqrt{3}} \sin \left(\frac{2}{3} \pi\right) \sin (x) \cos (y) \cos (z) \\
& v^{0}=\frac{2}{\sqrt{3}} \sin \left(-\frac{2}{3} \pi\right) \cos (x) \sin (y) \cos (z) \\
& w^{0}=0 \\
& \rho^{0}=1
\end{aligned}
$$

Periodic boundary conditions are applied in all spatial directions. Reynolds numbers of $\operatorname{Re}=800,1600,3000$ and resolutions of $N=64,128,256$ and 512 mesh points for each spatial direction were considered. The results were compared to DNS results of Brachet [4]. For adapting the time step according to the resolution we applied diffusive scaling in order to reconstruct the incompressible limit of the Navier-Stokes equations, see [34] among others. The numerical setups in terms of the lattice velocity $u_{L}$ as well as the Mach number $M a=u_{L} / c_{s}$ are listed in Table 1.

Table 1: Numerical setup for the Taylor-Green-Vortex at different resolutions.

\begin{tabular}{||c|c|c|c|c||}
\hline$N$ & $u_{L}$ & Ma & $\Delta x$ & $\Delta t$ \\
\hline \hline 64 & 0.1 & 0.1730 & 0.0981 & 0.00981 \\
\hline 128 & 0.05 & 0.0865 & 0.0490 & 0.00245 \\
\hline 256 & 0.025 & 0.0432 & 0.0245 & 0.000612 \\
\hline 512 & 0.0125 & 0.0216 & 0.0122 & 0.000152 \\
\hline
\end{tabular}

In the following, stability properties of the LBM schemes and their accuracy regarding the prediction of the temporal evolution of the integral energy dissipation rate computed from the resolved flow scales are discussed. In Fig. 1 the integral energy dissipation rate $\epsilon=\frac{d E(t)}{d t}$ for $R e=800$ is shown.

For the coarsest resolution of $N=64$ the MRT as well as the RLB scheme underestimate dissipation rates compared to the BGK scheme and the reference DNS data of Brachet [4]. When increasing the resolution to $N=128$, the evolution of the dissipation rate is slightly better recovered by the MRT approach compared to the BGK scheme. Again dissipation rates are always predicted lower by the RLB model compared to results of the BGK or the MRT scheme. For a resolution of $N=256$ only small differences between the three considered LBM schemes can be found, and at the highest resolution of $N=512$ at $R e=800$ they are in very good agreement with the reference DNS data. For $R e=1600$, see Fig. 2, 

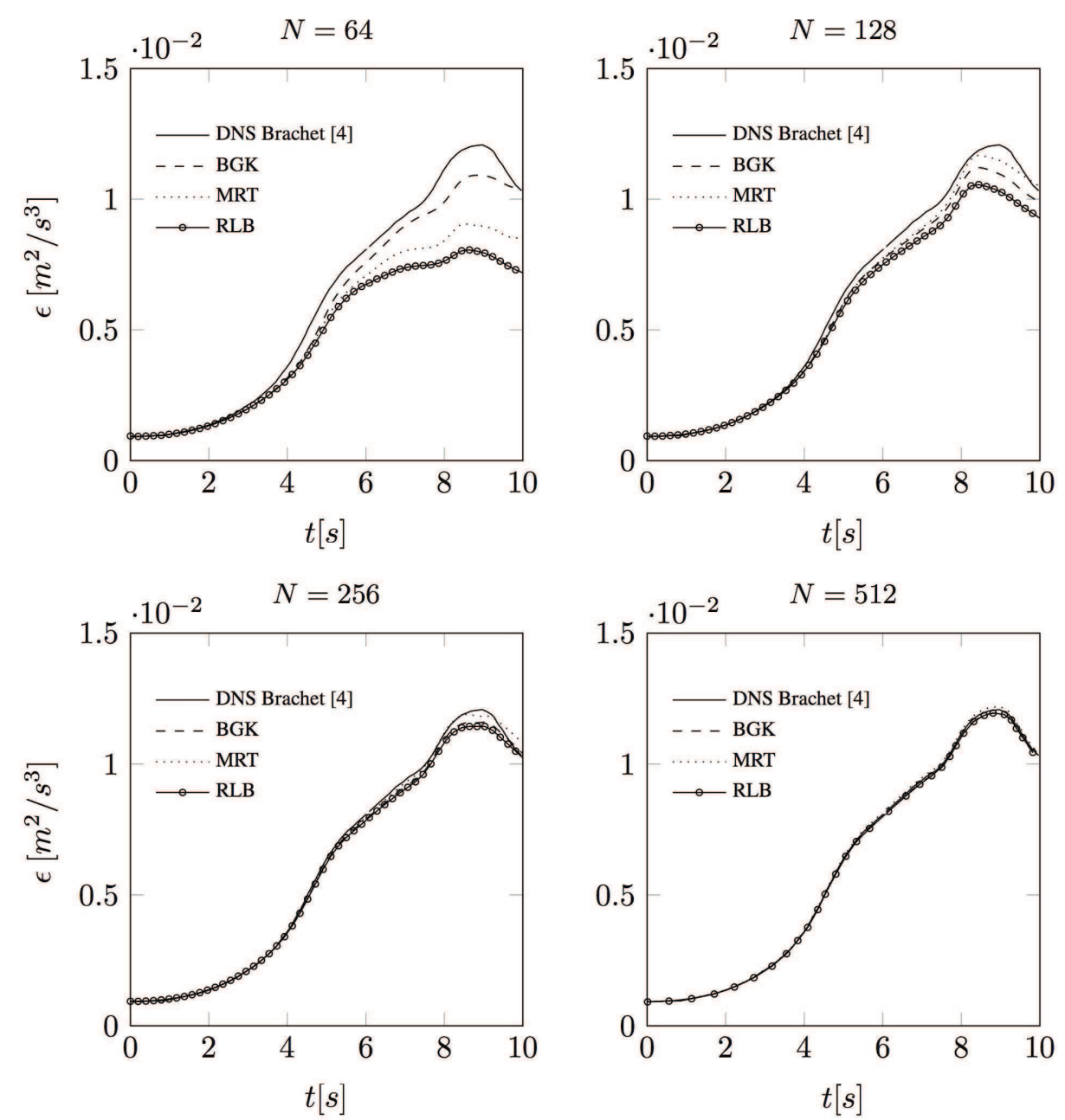

Figure 1: Temporal evolution of the dissipation rate of the Taylor-Green vortex predicted by the BGK, MRT and RLB scheme at $R e=800$ for the resolutions $N=64,128,256$ and 512 compared to DNS.

distinct differences between the schemes can be observed. When using the BGK scheme at the coarsest resolution of $N=64$ instabilities in the flow variables develop at $t \approx 5 s$, which lead to diverging simulations. Employing methods for a pressure initialization according to [6] did not improve the stability of the BGK scheme. It can be concluded, that these instabilities of the BGK scheme result from a too coarse resolution of the developing flow scales, which is similar to the characteristics of linear finite-difference schemes. In [26] a similar behavior of the BGK scheme had been observed. At the coarsest resolution, the MRT and the RLB scheme allow for stable simulations, however dissipation rates are predicted significantly lower compared to DNS reference data.

At an increased resolution of $N=128$ the flow scales developing during the simulation are sufficiently resolved for BGK scheme to remain stable and predict dissipation rates 

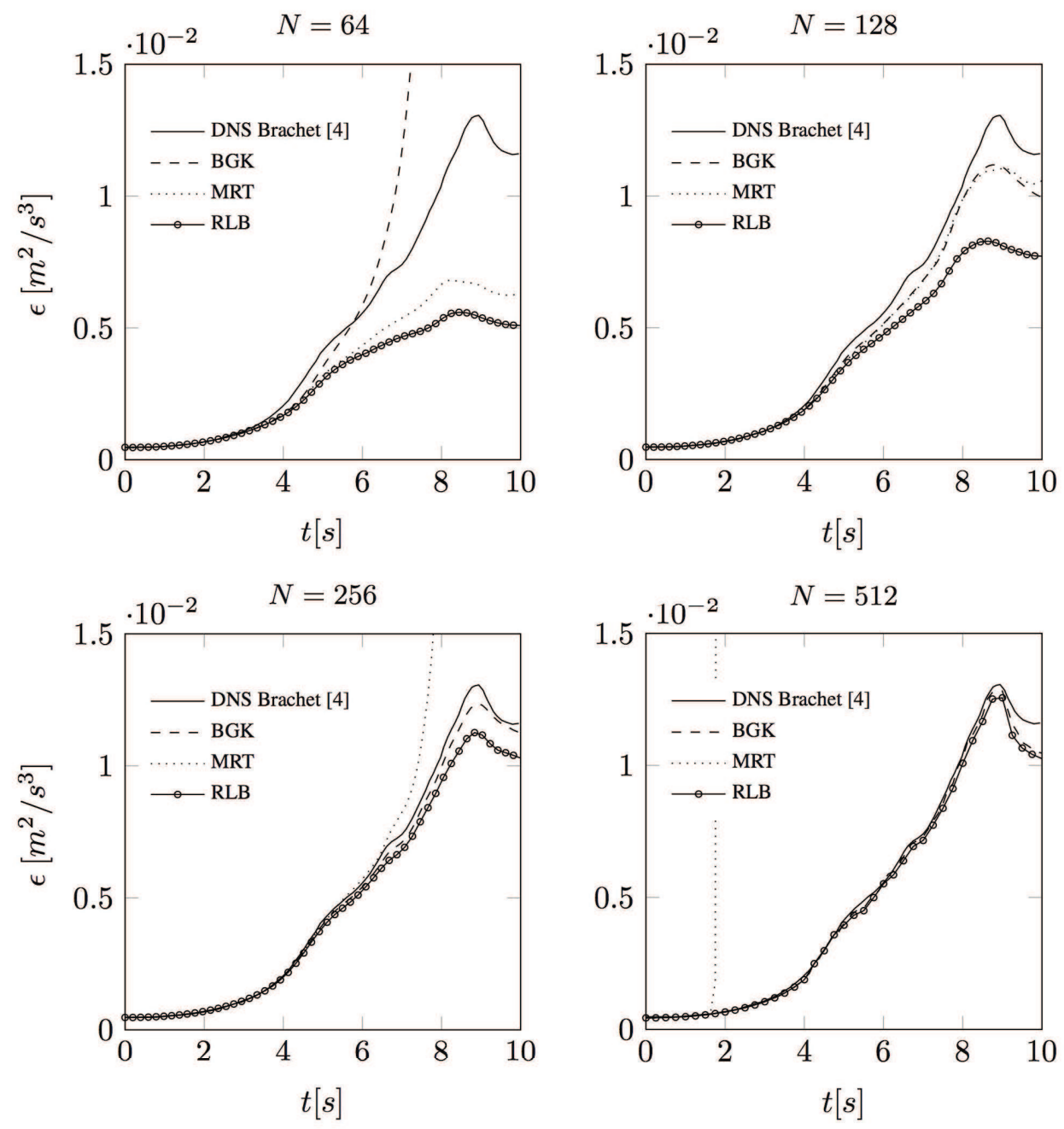

Figure 2: Temporal evolution of the dissipation rate of the Taylor-Green vortex predicted by the BGK, MRT and RLB scheme at $R e=1600$ for the resolutions $N=64,128,256$ and 512 compared to DNS.

with similar accuracy as with the MRT scheme. Again, the RLB scheme captures the peak dissipation rate at $t \approx 9 \mathrm{~s}$ but predicts its magnitude by approximately $38 \%$ too low with respect to the DNS reference data, and by approximately $23 \%$ too low with respect to the BGK and MRT results. When increasing the resolution further to $N=256$ the accuracy of the BGK and of the RLB scheme improves, whereas the MRT scheme exhibits instabilities from $t \approx 7 s$ onwards. In an attempt to stabilize the simulations a pressure initialization according to [6] was carried out, which however did not alter the observed behavior of the MRT scheme. At the highest resolution of $N=512$ the BGK and the RLB scheme showed good agreement with reference DNS data and only after the peak of the dissipation rate at $t \approx 9 \mathrm{~s}$ notable differences become visible. At $N=512$ the MRT scheme 

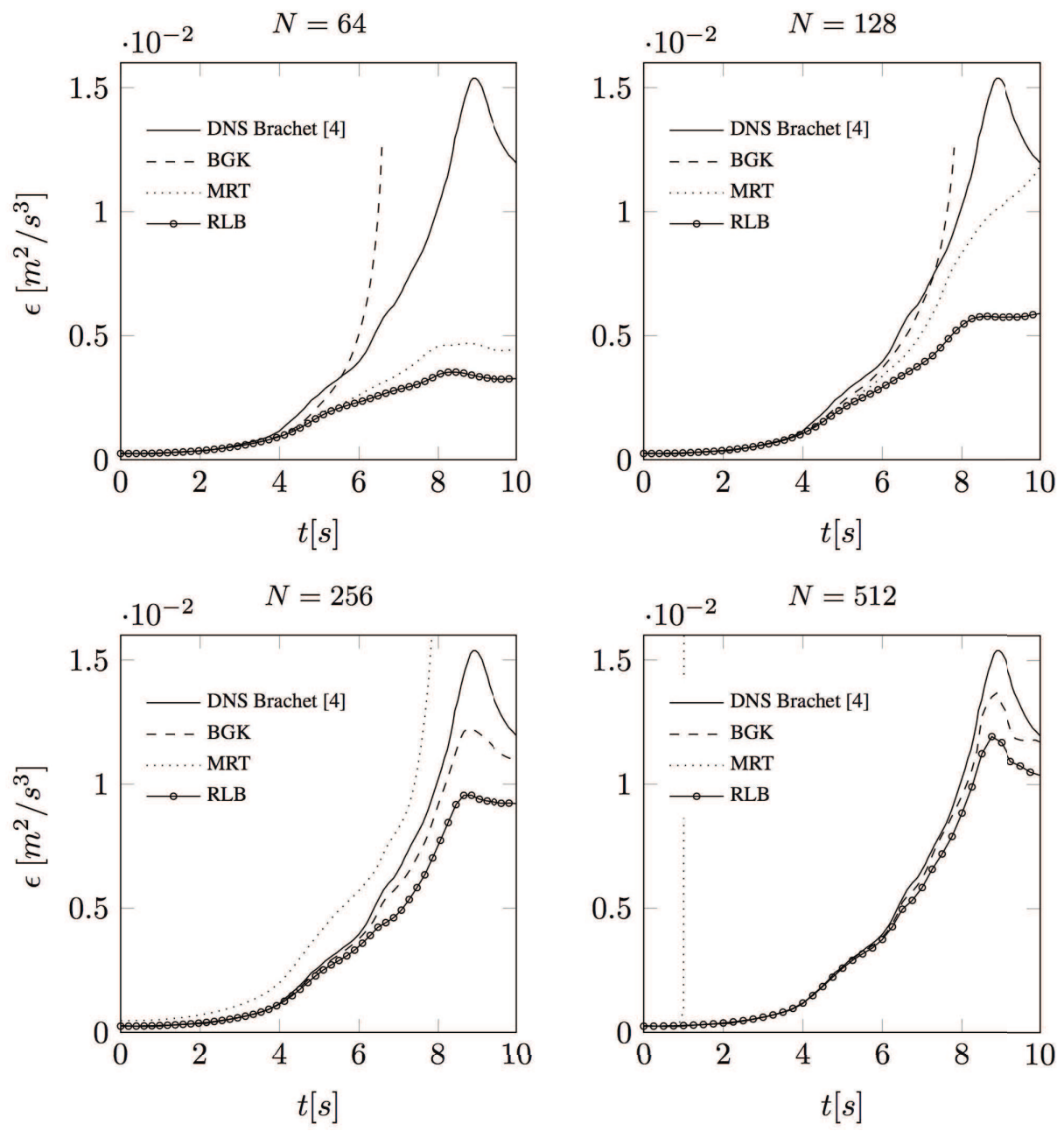

Figure 3: Temporal evolution of the dissipation rate of the Taylor-Green vortex predicted by the BGK, MRT and RLB scheme at $R e=3000$ for the resolutions $N=64,128,256$ and 512 compared to DNS.

exhibits instabilities at $t \approx 1.5 \mathrm{~s}$, which is significantly earlier compared to the simulation at $N=256$, and the simulation diverges again.

The same tendencies found before for both lower Reynolds numbers, also prevail at $R e=3000$, see Fig. 3 . The RLB scheme again is stable for all resolutions considered, yet dissipation rates are less accurate compared to successful simulations employing the BGK or the MRT scheme. The MRT scheme allows for stable simulations only when using a strongly under-resolved setup with $N=64$ mesh cells in each direction at this Reynolds number of $R e=3000$. For the resolutions $N=256,512$ the MRT simulations diverged and by employing $N=128$ cells in each direction the solution converged towards an unphysical state for $t>10 \mathrm{~s}$. Using the BGK scheme stable and reasonable accurate simulations where performed for mesh resolutions of $N=256$ and $N=512$. 
The distinct differences between the schemes with respect to mesh resolution and Reynolds number found so far are summarized as follows:

- The BGK scheme allows for stable simulations in case flow scales are sufficiently resolved by the employed mesh. Yet, a criterion to quantify this Reynolds number dependent resolution requirement for turbulent flows has not been found so far. For increasing mesh resolution an increasing accuracy and hence mesh convergence can be obtained. If the turbulent flow scales are not sufficiently resolved, non-linear instabilities were found to develop, which is in agreement with previous observations, e.g., Luo and Lallemand [26].

- The MRT scheme shows good results regarding the integral energy dissipation rates for under-resolved DNS setups. For increasing resolutions, accuracy improved for the lowest Reynolds number of $R e=800$ considered here. However, for increased resolutions at higher Reynolds numbers of $R e=1600,3000$ non-linear instabilities became apparent and simulations diverged. The latter finding seems to prevent the MRT scheme to obtain mesh convergence when used in a DNS setting.

- The RLB scheme has been found to perform stable simulations at all Reynolds numbers and resolutions considered here. Yet it suffers from the largest numerical dissipation compared to the other schemes.

For further analysis of the behavior of the LBM schemes, three-dimensional energy spectra $E(\xi, t)=\frac{1}{2}<\hat{u}(\xi, t) \hat{u^{*}}(\xi, t)>$ for all three Reynolds numbers, different spatial resolutions and at two instants in time, $t=6 \mathrm{~s}$ and $t=8 \mathrm{~s}$, are considered in the following. Here $\hat{u}(\xi, t)$ is the complex Fourier transform of the velocity field and $\hat{u}^{*}(\xi, t)$ is the complex conjugate respectively [40]. In Fig. 4, energy spectra at $R e=800$ for the resolutions $N=64,128,256$ are shown. When employing the BGK scheme at the coarsest resolution $N=64$ significantly large energy levels are present at the highest wave numbers for both instants in time $t=6 \mathrm{~s}$ and $t=8 \mathrm{~s}$. For this resolution, the spectra of the BGK scheme do not show the expected strong decrease of energy in the dissipative range at the highest wavenumbers, whereas the MRT as well as the RLB scheme exhibit this characteristic. Therefore, the more accurate prediction of the energy dissipation rate of the BGK scheme for $N=64$ compared to the MRT and RLB scheme, see Fig. 1, is due to this unphysical effect in the high wave number range. If the resolution is increased to $N=128$ and $N=256$, this accumulation of energy at high wave numbers when using the BGK scheme is reduced, see Fig. 4 . For $N \geq 256$ all three LBM schemes show very similar energy spectra and a short inertial as well as a dissipative range can be observed.

In Fig. 5 energy spectra are shown for $R e=1600$. For the BGK scheme again increased energy levels at high wave numbers can be observed in case flow structures are not sufficiently resolved by the computational mesh. This behavior leads to a diverging simulation using a resolution of $N=64$ for $t>6 s$, see Fig. 5. The spectrum shows a large accumulation of energy in the highest dissipative wave numbers, which changes the slope of $E(\xi)$ in this region being even larger than the well known slope of $-5 / 3$ for the inertial 

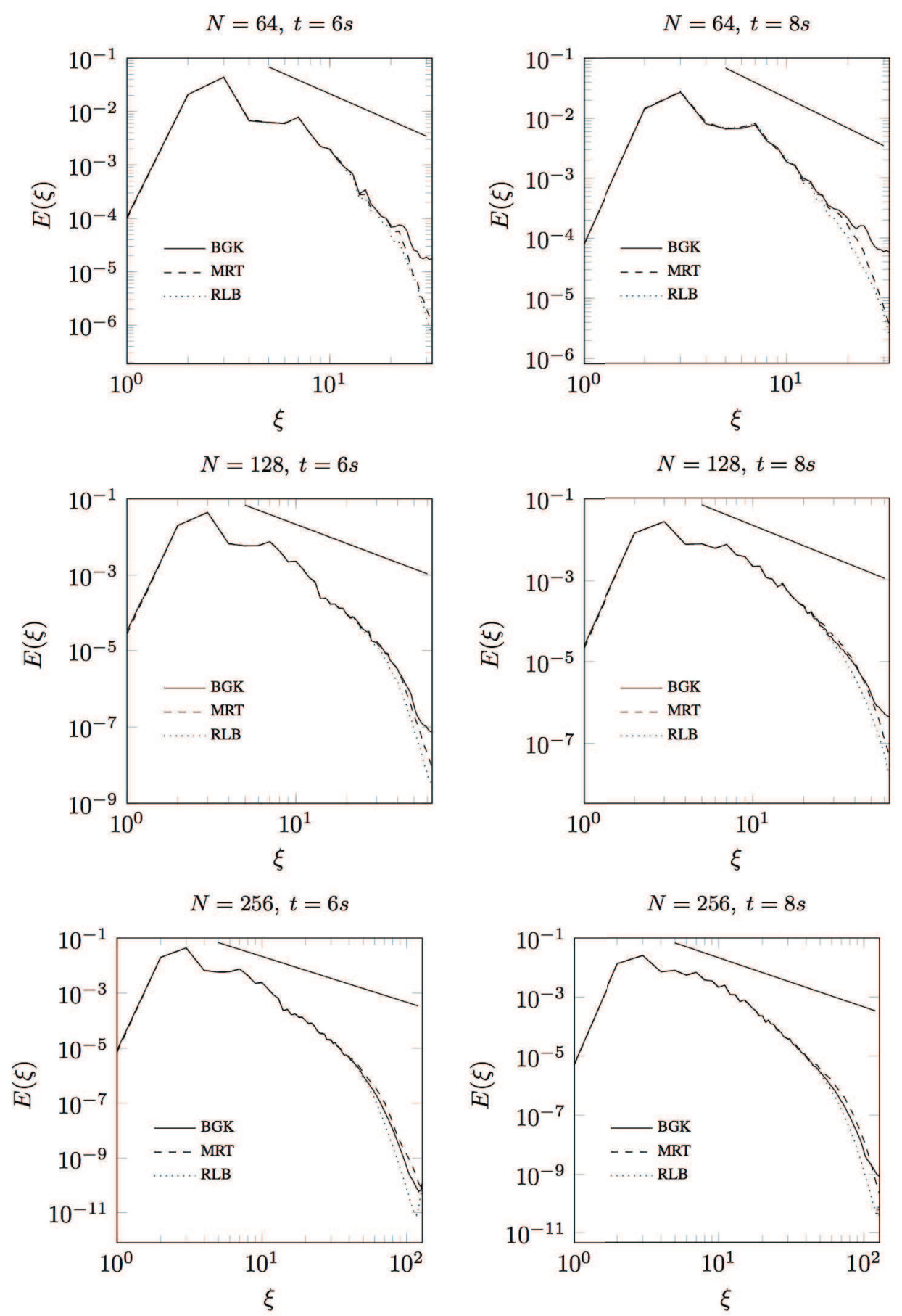

Figure 4: Energy spectra of the Taylor-Green vortex for different times and resolutions at $R e=800$ for the BGK, MRT and RLB scheme and a straight line of slope $-\frac{5}{3}$ being characteristic for the inertial range of homogeneous isotropic turbulence. 

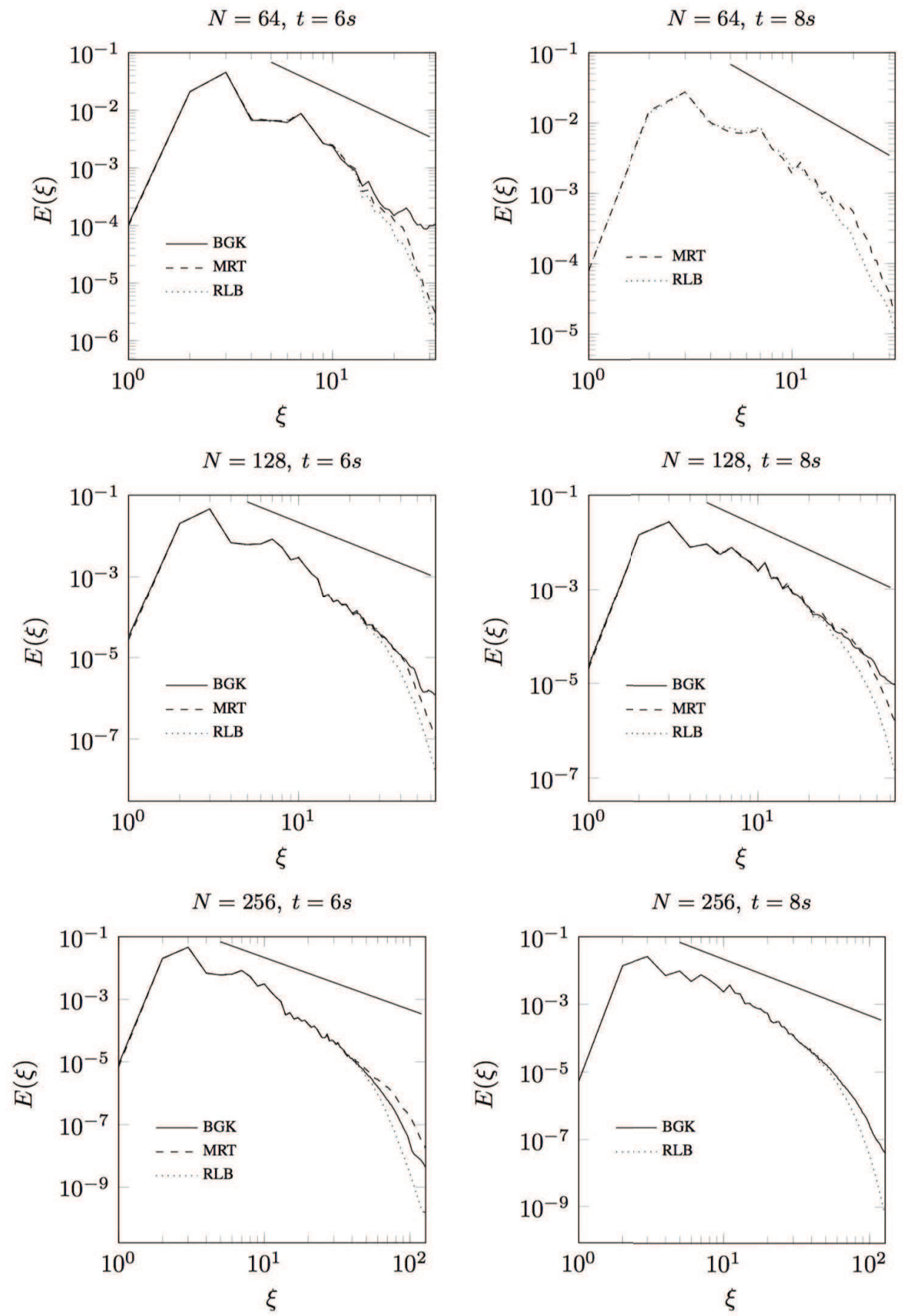

Figure 5: Energy spectra of the Taylor-Green vortex for different times and resolutions at $R e=1600$ for the BGK, MRT and RLB scheme and a straight line of slope $-\frac{5}{3}$ being characteristic for the inertial range of homogeneous isotropic turbulence. 
range. At $t=8 \mathrm{~s}$ the simulation of the Taylor-Green vortex at $R e=1600$ using the BGK scheme and $N=64$ already diverged, so no graph is shown in the corresponding figure. In case the resolution is increased to $N=128$ the BGK scheme still exhibits increased energy levels in the highest wave numbers, yet the simulations remain stable and integral dissipation rates of acceptable accuracy where obtained. For resolutions $N \geq 256$ spectra of the BGK scheme feature distinct inertial and dissipative wave number ranges. Using the MRT scheme for simulations at $R e=1600$ reasonable results were obtained for low resolutions. However for $N \geq 256$ the simulations diverged. The reason for this behavior can bee seen in Fig. 5 at $N=256, t=6 s-8 s$ : The MRT scheme exhibits unphysical large energy levels in the highest wave number region, which subsequently lead to the development of instabilities and a break-down of the method well before $t=8 \mathrm{~s}$. This finding is similar to that for the BGK scheme in under-resolved setups, whereas for the MRT scheme this accumulation of energy occurs for well-resolved setups. For the RLB scheme an inertial as well as a dissipative region can be observed in all spectra considered. However, the onset of the dissipative range, i.e. the strong decrease of kinetic energy, takes place always at significantly lower wave numbers compared to the BGK and the MRT scheme. This pronounced reduction of energy in the high wave number range allows for stable simulations, but it is also the reason for the strong numerical dissipation which impairs the accuracy of integral dissipation rates as seen in Figs. 1-3. The obtained energy spectra for the highest Reynolds number of $R e=3000$, see Fig. 6, confirm the previously found behavior of the considered LBM schemes. The RLB scheme allows for stable simulations at all resolutions considered, but exhibits high numerical viscosity and consequently low mesh convergence rates. The BGK scheme requires resolutions of $N \geq 256$ for converging simulations of the Taylor-Green vortex at $R e=3000$. At $N=256$ unphysical energy levels at high wave numbers develop at later times $t \geq 8 \mathrm{~s}$, however these effects can still be tolerated by the scheme and do not lead to instabilities yet. For the MRT scheme the opposite behavior is found again: Only for the very coarse resolution of $N=64$ of TaylorGreen vortex at $R e=3000$ stable computations are obtained. Of course, the accuracy with respect to dissipation rates is rather low as expected. For increased resolutions of $N=128$ and $N=256$ the simulations became unstable after $t=8 \mathrm{~s}$ and $t=2 \mathrm{~s}$, respectively.

For further investigation of the observed instabilities of the BGK as well as the MRT scheme at $R e=3000$ and a spatial resolution of $N=256$ the temporal evolution of single modes of the dissipation spectra $\epsilon(\xi, t)=\frac{d E(\xi, t)}{d t}$ are considered, see Fig. 7. The reference data of Brachet [4] on the temporal evolution of the integral dissipation rate at this Reynolds number shows two characteristic changes before reaching its peak value at $t \approx 9$ s, see Fig. 3: At $t \approx 4 \mathrm{~s}$ the growth rate of the dissipation rate increases and at $t \approx 6 \mathrm{~s}$ again a further increase of the slope of the dissipation-rate curve can be observed. When considering the modes of the dissipation rate, Fig. 7, for both the BGK and the MRT scheme the lower modes $\xi=1, \cdots, 9$ are smoothly increasing starting at $t=0$ s until $t \approx 6 \mathrm{~s}$, hereafter only slight changes are notable. However the dissipation rate magnitudes of higher modes $\xi=83, \cdots, 127$ initially remain very low. At $t \approx 2 s$ a strong growth of the higher dissipation modes can be observed featuring a steep slope, which starts to flatten 

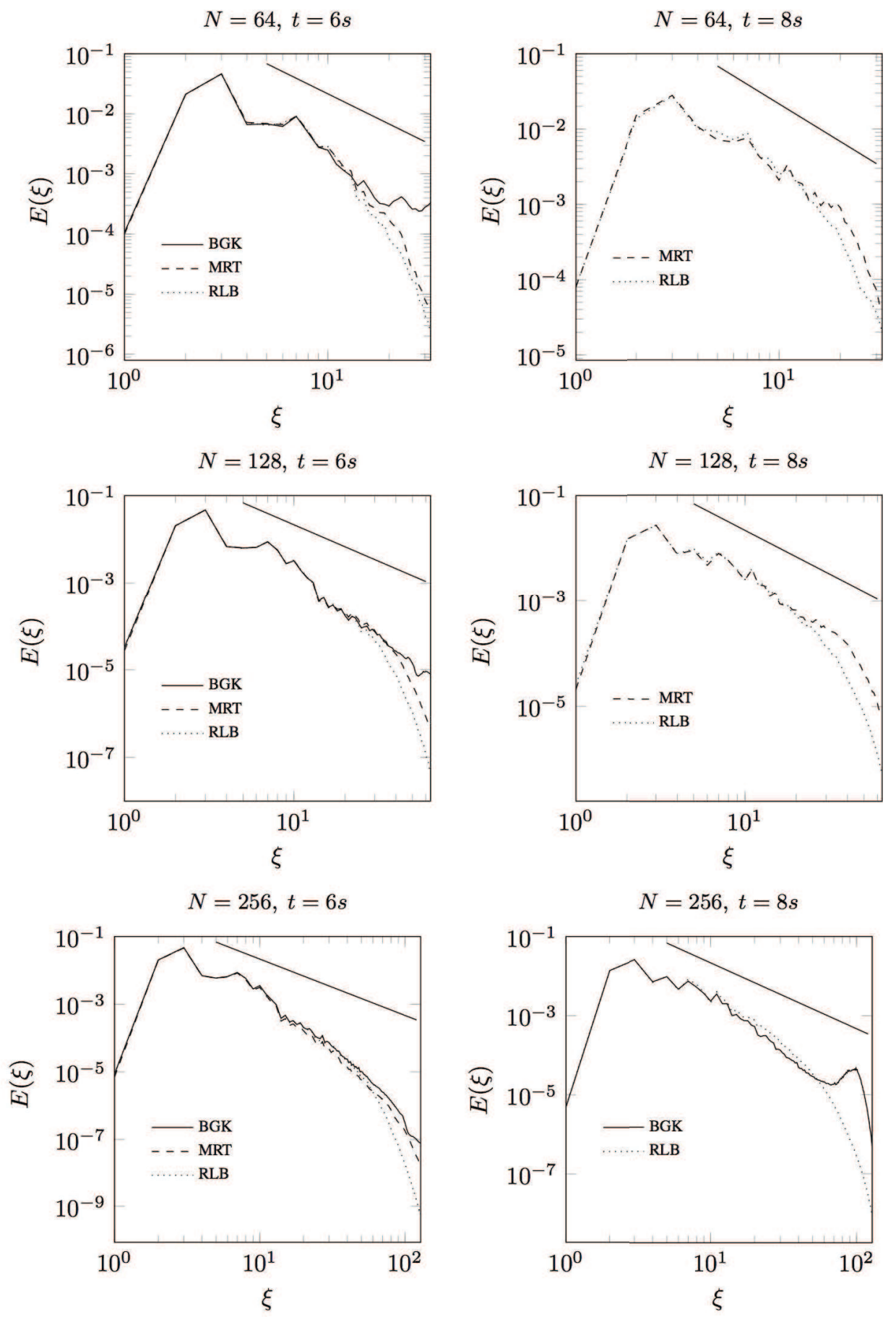

Figure 6: Energy spectra of the Taylor-Green vortex for different times and resolutions at $R e=3000$ for the BGK, MRT and RLB scheme and a straight line of slope $-\frac{5}{3}$ being characteristic for the inertial range of homogeneous isotropic turbulence. 
(a) BGK

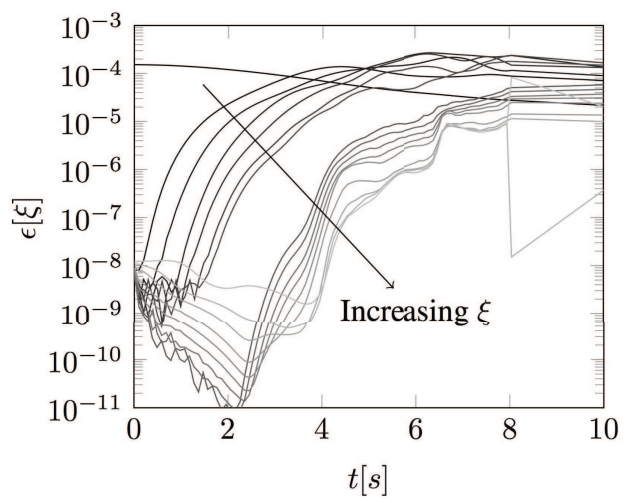

(b) MRT

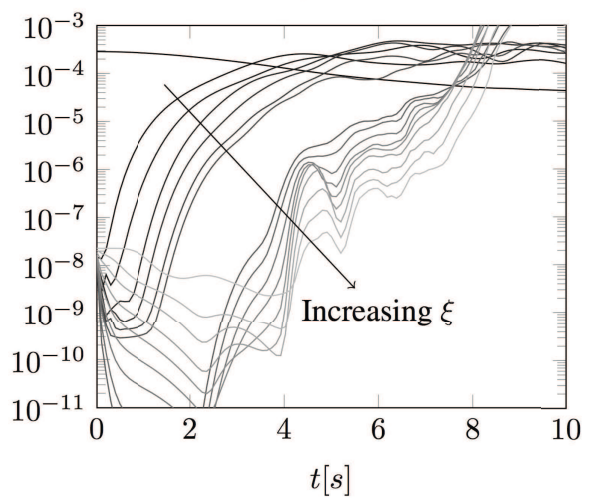

Figure 7: Temporal development of dissipation modes of the Taylor-Green vortex for the BGK and MRT scheme at $R e=3000$ and $N=256$.

only after $t \approx 4 s$. At this instant in time, also the resulting first increase of the integral dissipation rate in Fig. 3 can be identified. For the BGK scheme this growth of the higher modes is accomplished rather smoothly without notable oscillations in the growth rates. For the MRT scheme however, following the initial strong growth of the higher modes a significant change of the growth rate can be observed for $t \geq 4.5 \mathrm{~s}$, where even negative slopes can be found. This leads to oscillations in the magnitude of the higher modes, which subsequently are reduced in amplitude, but are still visible at later times, e.g. $t \approx 6 \mathrm{~s}$. At $t \approx 7 \mathrm{~s}$ a further increase especially of the highest dissipation modes can be observed for the BGK scheme, Fig. 7(a). This corresponds to second steepening of the slope of the integral dissipation rate as seen in Fig. 3. Thereafter the magnitude of the highest dissipation modes for the BGK scheme change only slightly with two exceptions: For $\xi=120$ a large increase by approximately one order of magnitude can be observed and for the mode $\xi=127$ an even larger decrease by three orders of magnitude can be identified at $t \approx 8$ s. This is attributed to an instability of the BGK scheme in case flow structures are not sufficiently resolved by the underlying mesh. The result of this instability can also be seen in the energy spectrum for $R e=3000, N=256$, see Fig. 6, where for the instant in time $t=8 \mathrm{~s}$ unphysical large energy levels at high wave numbers are present. Despite these instabilities, the BGK scheme allowed for converging simulation using this setup. This was not the case for the MRT scheme. In Fig. 7(b) a sudden increase of the three dissipation modes $\xi=113,120$ and 127 by approximately four orders of magnitude indicate a large unphysical energy increase of these modes. Also the modes $\xi=90,97$ and 104 are found to grow continuously, which is unphysical and lead to an unstable simulation.

\section{Wall Bounded Turbulence: The Turbulent Channel Flow}

In this section the BGK, MRT and RLB schemes are tested for the turbulent channel flow at three different Reynolds friction numbers $\left(R e_{\tau}=180, R e_{\tau}=395\right.$ and $\left.R e_{\tau}=590\right)$ for 
varying resolutions. Results shall outline if the conclusions drawn for the homogenous isotropic turbulence test case, can be transferred to wall-bounded turbulence. In this section, we do not provide detailed mesh-convergence studies, but rather show results obtained by varying the resolution at fixed Reynolds numbers as done in the previous section.

Our numerical test case is based on the work of Bespalko [2] who performed a detailed grid convergence study for the turbulent channel flow by applying the BGK scheme. The domain has the extensions of $L_{x}=12 \mathrm{H}, L_{z}=4 \mathrm{H}$ and $L_{y}=2 \mathrm{H}$ in streamwise, lateral and wall-normal direction, respectively, with $H$ being the channel half width. In streamwise and lateral direction, periodic boundary conditions were applied. In order to represent the upper and lower channel wall, an extended finite difference boundary condition, outlined by Latt et al. [30], is employed. Due to the abundance of populations propagating from the wall into the bulk flow, all populations in the wall cells are reconstructed to conserve density $\rho$ and momentum $\rho \boldsymbol{u}$ and to calculate the stress tensor $\sigma_{i j}$. The stress tensor is obtained by relating it to the strain rate tensor, which is constructed by finite differences of the velocity in the neighboring cells. Latt et al. [30] showed that this boundary condition yields numerical stability and accuracy of second order, which makes it suitable for the simulation of flows at high Reynolds numbers. In contrast to other boundary conditions, representing solid no-slip walls, the wall nodes are fixed on the wall leading to a constant cell size and wall distance $\Delta x=\Delta y=\Delta z$.

Resolution is varied in a similar way as for the Taylor-Green vortex, leading to resolved and under-resolved test cases. Adjusting the friction Reynolds number is achieved by applying diffusive scaling. The viscosity is changed according to the Reynolds number and the lattice velocity according to the resolution. To estimate the viscosity we use the Dean correlation, see [33]

$$
\operatorname{Re}_{B}=\left(\frac{8}{0.073}\right)^{4 / 7} R e_{\tau}^{8 / 7}
$$

which connects the bulk Reynolds number $R e_{B}$ to the friction Reynolds number $R e_{\tau}$. With $\operatorname{Re}_{B}=\frac{u_{B} L_{z}}{v}$, we can rewrite Eq. (4.1) as

$$
v=\frac{u_{B} L_{z}}{\left(\frac{8}{0.073}\right)^{4 / 7} R e_{\tau}^{8 / 7}} .
$$

Based on the work of Bespalko [2], we set $u_{B}=0.111 \frac{\mathrm{m}}{\mathrm{s}}$. To account for the limited validity of the Dean correlation at low Reynolds numbers, the viscosity for $R e_{\tau}=180$ is obtained by a virtual log law which spans from the wall to the channel half height as described in [2]

$$
v=\frac{u_{B} L_{z}}{2 \operatorname{Re}_{\tau}\left(\frac{1}{\kappa} \ln \left(\operatorname{Re}_{\tau}\right)+A\right)} .
$$

A full list of conducted simulations with the final physical state, lattice velocities $u_{L}$, resolution $N$ and Mach number is given in Table 2. 
Table 2: Performed simulations for the Turbulent Channel Flow.

\begin{tabular}{||c|c|c|c|c|c|c||}
\hline Model & $N$ & $\Delta y^{+}$ & $R e_{\tau, \text { target }}$ & $R e_{\tau, \text { real }}$ & $u_{L}$ & Ma \\
\hline \hline BGK & 31 & $\approx 5.8$ & 180 & - & 0.111 & 0.1902 \\
\hline BGK & 91 & 2.065 & 180 & 188.81 & 0.0378 & 0.0654 \\
\hline MRT & 31 & $\approx 5.8$ & 180 & - & 0.111 & 0.1902 \\
\hline MRT & 91 & 1.87 & 180 & 177.32 & 0.0378 & 0.0654 \\
\hline RLB & 31 & $\approx 5.8$ & 180 & - & 0.111 & 0.1902 \\
\hline RLB & 91 & - & 180 & 55.97 & 0.0378 & 0.0654 \\
\hline BGK & 91 & 4.031 & 395 & 389.97 & 0.0378 & 0.0654 \\
\hline BGK & 151 & 2.615 & 395 & 394.865 & 0.0227 & 0.0394 \\
\hline MRT & 91 & 3.705 & 395 & 363.02 & 0.0378 & 0.0654 \\
\hline MRT & 151 & 2.383 & 395 & 359.75 & 0.0227 & 0.0394 \\
\hline BGK & 91 & $\approx 6.48$ & 590 & - & 0.0378 & 0.0654 \\
\hline BGK & 151 & 3.981 & 590 & 589.91 & 0.0227 & 0.0394 \\
\hline MRT & 91 & $\approx 6.48$ & 590 & - & 0.0378 & 0.0654 \\
\hline MRT & 151 & $\approx 3.907$ & 590 & - & 0.0227 & 0.0394 \\
\hline
\end{tabular}

Since the variation of the resolution leads to different wall distances in terms of the non-dimensional $y^{+}=\frac{u_{\tau} \Delta z}{v}$ value, the evaluation of the skin friction velocity $u_{\tau}$ is different. For properly resolved setups, i.e. $\Delta y^{+}<5, u_{\tau}$ can be calculated as $u_{\tau}=\sqrt{\frac{\tau_{w}}{\rho}}$ with $\tau_{w}$ being the wall shear stress. Configurations with $\Delta y^{+}>5$ we use Eq. (4.1) for calculating $u_{\tau}$. The different resolutions with the corresponding target and calculated $R e_{\tau}$ are listed in Table 2 as well. Note that proper $\Delta y^{+}$values for the MRT model at $R e_{\tau}=590$ are not given since the simulations reproduced an unphysical state. This will be discussed later in this section. The RLB model did not achieve a turbulent state at $R e_{\tau}=180$ with $N=31$ and $N=91$, leading to friction Reynolds numbers which indicate laminar flow, see also [42].

The target mass flow is imposed by applying adaptive forcing according to the work of Cabrit [5]. The volume force is computed as

$$
g=\frac{u_{\tau}^{2}}{N}+\left(u_{B}-\left\langle u_{x}\right\rangle\right) \frac{u_{B}}{N}
$$

where $u_{B}$ is the bulk velocity, $\left\langle u_{x}\right\rangle$ the instantaneous space average of the streamwise velocity component and $g$ the resulting volume force. The force is included into the LBM schemes by employing the approach of He et al. [19] for the BGK and RLB scheme. For the MRT scheme we make use of the forcing approach of Ladd and Verberg [25].

A proper initialization of the velocity field and triggering of turbulence is needed in order to reach the desired turbulent state. We employ a $1 / 7$ power law i.e. $u(z)=$ $u_{\text {char }}(z / H)^{\frac{1}{7}}$, which is superimposed with statistically random perturbations $u^{\prime}, v^{\prime}$ and 
$w^{\prime}$ drawn from a normal distribution $\frac{1}{\sigma \sqrt{2 \pi}} e^{-\frac{r-\mu}{2 \sigma^{2}}}$. Hereby $r$ is a random number between -1 and $1, \mu=0$ is the mean and $\sigma$ the standard deviation, which was set to $5 \%$.

Statistics are obtained for all schemes in the same way to have a consistent comparison of the applied models. Averaging is performed spatially over horizontal planes in the homogeneous directions and temporally after the flow was uncorrelated. Based on the work of Moser [35] the flow is weakly correlated $(\approx 0.08)$ at a streamwise distance of $2 \mathrm{~m}$. This leads to a sampling time of $\Delta t=18.01 \mathrm{~s}$ with $u_{B}=0.111 \frac{\mathrm{m}}{\mathrm{s}}$. After a physical simulation time of $t=163.12 s$, we continue averaging over a time period of $\Delta t=8596$ s, i.e. 79.5 flow through times for $R e_{\tau}=180$. For increasing $R e_{\tau}$, the correlation decreases, yet we kept the sampling time constant and increased the flow through times according to the increase of the turbulent time scale. By assuming the ratio of the large- and small-time turbulence scale behaves as $\frac{t_{L}}{t_{\eta}}=\sqrt{R e_{\tau}}$, see [36], the flow through times increased to 117.7 and 143.9 for $R e_{\tau}=395$ and $R e_{\tau}=590$ respectively.

\subsection{Convergence analysis of the turbulent channel flow}

Before mean statistics are shown, a visualization of the unsteady flow field is given and the statistical convergence of the simulations is demonstrated. For increasing Reynolds number the BGK approach consistently predicts an increasing number of small scale flow structures, see Fig. 8. When the MRT scheme is employed, only for the lowest Reynolds number $R e_{\tau}=180$ a reasonable flow field can be obtained, see Fig. 9. For $R e_{\tau}=395$ and $R e_{\tau}=590$, strong unphysical oscillations develop. The presence of side walls and the viscous damping in these regions presumably prevents instability of the simulations, yet the results are not physically meaningful.

The RLB scheme is not shown in this context, since we found it to re-laminarize for $N=31$ and $N=91$ at $R e_{\tau}=180$. Strong numerical dissipation, which already lead to under predicted dissipation rates in the previous section, apparently damps the initial disturbances, which in turn should trigger the flow to a turbulent state. Increasing the resolution from $N=31$ to $N=91$ amplifies this effect, since the imposed fluctuations are now distributed across a larger range of wavenumbers. Consequently, for an equal amount of kinetic energy that is associated to the fluctuations, the relative kinetic energy per wavenumber is decreased. Since we did not employ better initialization techniques or restarted a RLB simulation from a converged BGK solution we omit further investigations of the RLB model for $R e_{\tau}=395$ and $R e_{\tau}=590$. The convergence of the Reynolds friction number $R e_{\tau}=180$ during the initial transition phase is depicted in Fig. 10 for $N=91$.

In Fig. 11 we demonstrate the symmetry for the streamwise Reynolds stresses $\overline{u^{\prime} u^{\prime}}$ at $R e_{\tau}=180$. For both, MRT as well as the BGK scheme, the differences in the peak value is below 3\%. Although one could argue that this value leads to poorly-converged statistics, the symmetry across the channel height is satisfied to be more than $99.5 \%$ for both schemes. Consequently we can assume that, $(i)$ the flow statistics are practically converged and (ii) since for all simulations outlined, the overall discrepancies in terms 

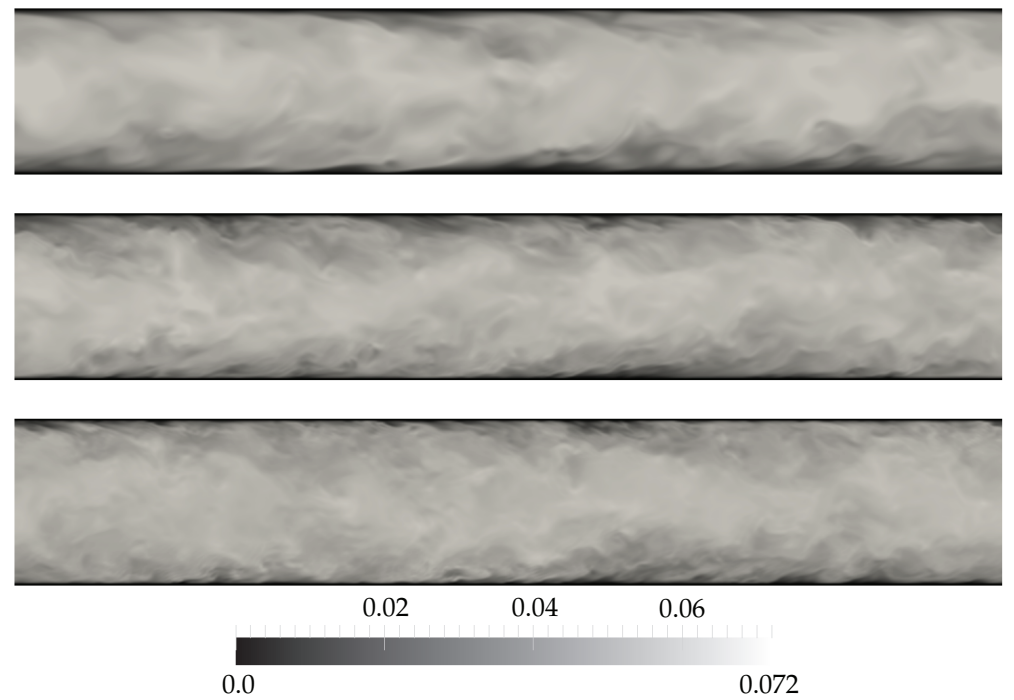

Figure 8: Instantaneous velocity magnitude in $\mathrm{m} / \mathrm{s}$ on a $x z$-plane located at $y=0.5 L_{y}$ predicted by the BGK collision model. $R e_{\tau}=180,395$ and 590 from top to bottom.
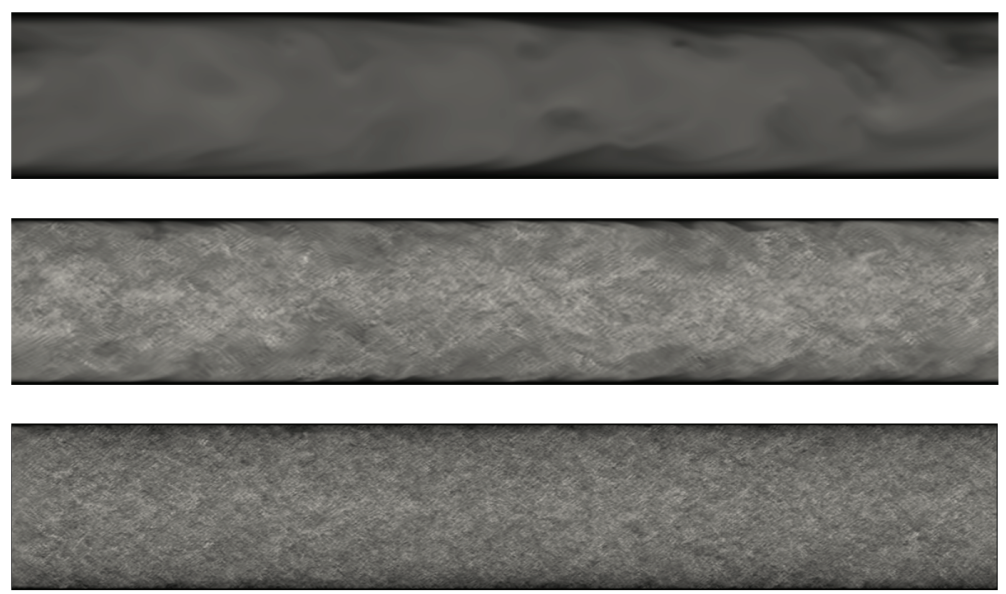

$\begin{array}{lll}0.025 & 0.05 & 0.075\end{array}$

0.0

0.10

Figure 9: Instantaneous velocity magnitude in $\mathrm{m} / \mathrm{s}$ on a $x z$-plane located at $y=0.5 L_{y}$ predicted by the MRT collision model. $R e_{\tau}=180,395$ and 590 from top to bottom.

of the peak value prediction between the different numerical schemes is higher than the error in the statistics, the interpretation of the results is not affected by the averaging procedure. 


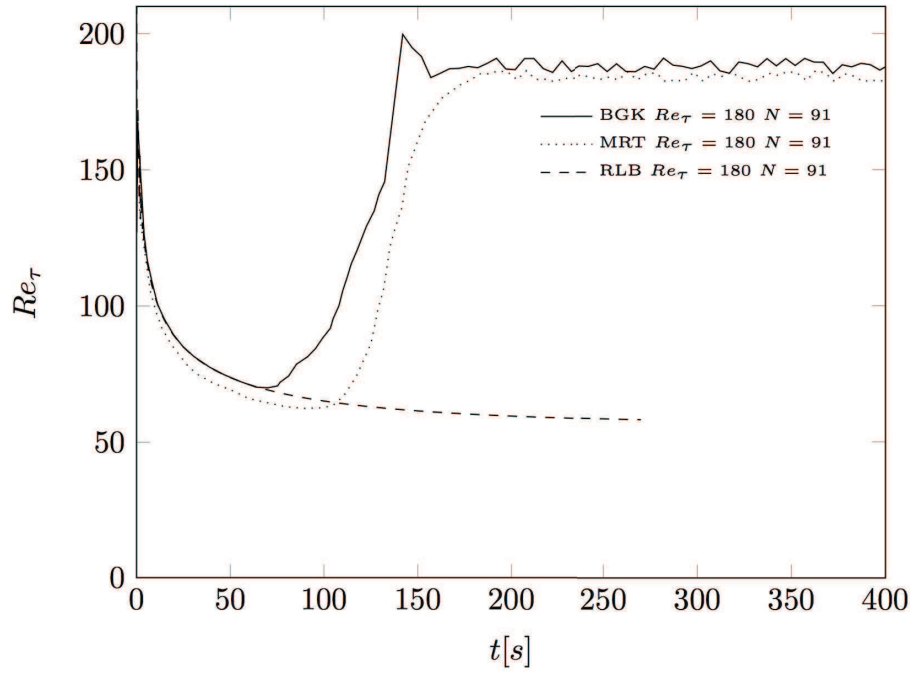

Figure 10: Convergence of $R e_{\tau}$ as a function of time for $R e_{\tau}=180$ and $N=91$.

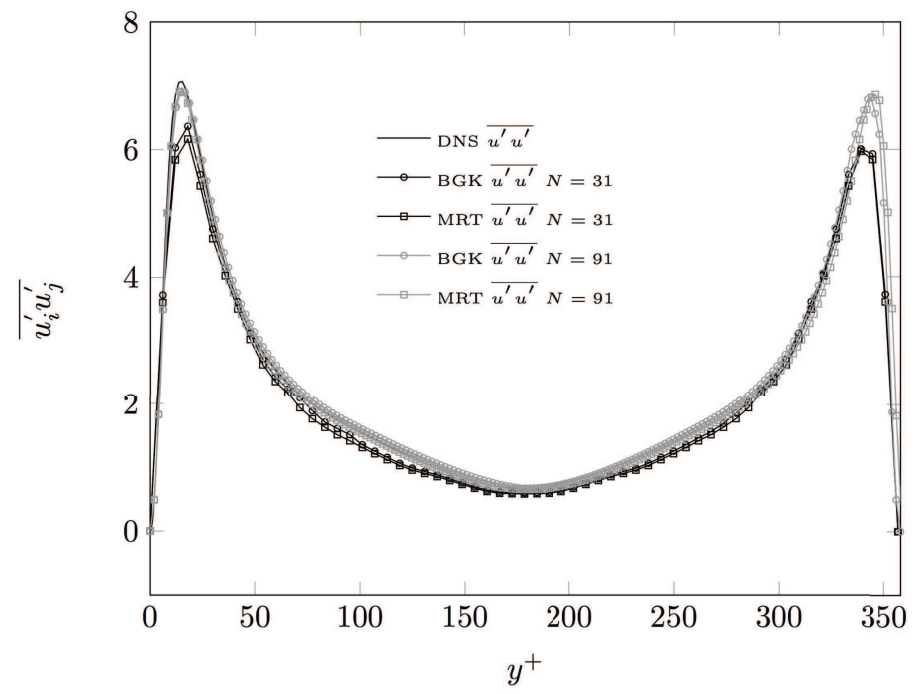

Figure 11: Symmetry proof for the streamwise Reynolds stress at $R e_{\tau}=180$ for $N=31$ and $N=91$ compared to the DNS results of Kim et al. [22]. For the reference DNS only data for the channel half is provided.

\subsection{Mean velocity statistics}

In Fig. 12 the mean velocity profiles for $R e_{\tau}=180$ from DNS of Kim et al. [22] are shown along with the under-resolved simulations using the BGK, MRT and RLB schemes. For $y^{+}<=10$ the reference DNS and the under-resolved simulations of the BGK and MRT scheme are in fair agreement. In the logarithmic region an increased normalized velocity 


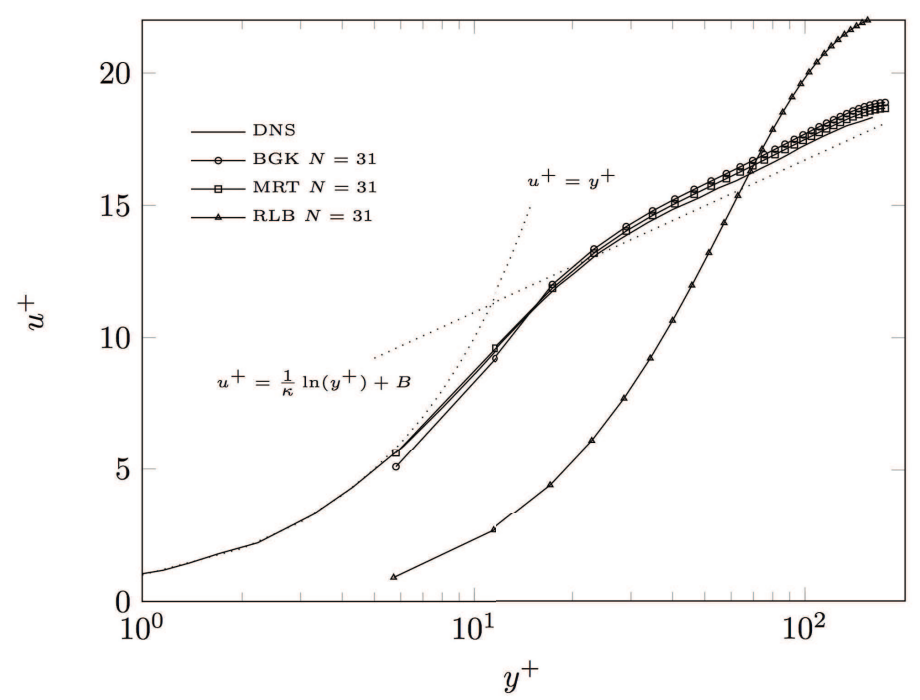

Figure 12: Mean velocity profiles of turbulent channel flow at $R e_{\tau}=180$ and $N=31$ compared to the DNS of Kim et al. [22].

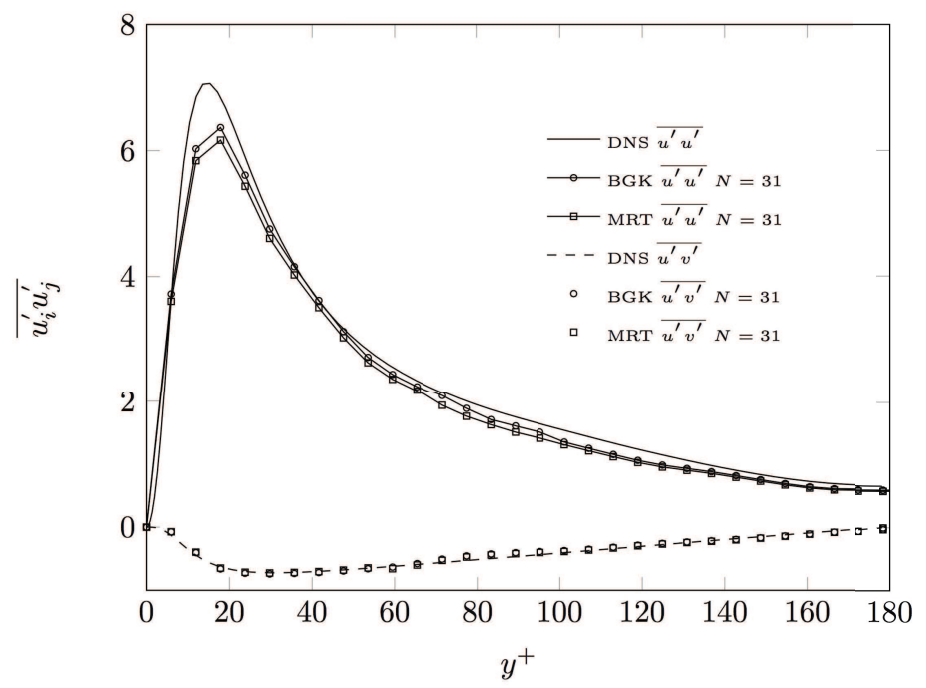

Figure 13: Mean Reynolds stresses of turbulent channel flow at $R e_{\tau}=180$ and $N=31$ compared to the DNS of Kim et al. [22].

$u^{+}=\frac{\bar{u}}{u_{\tau}}$ by $\approx 5 \%$ for the BGK simulation and by approximately $3 \%$ for the MRT simulation can be found. The average velocity profile of the RLB scheme corresponds to a laminar flow profile. The employed resolution is, compared to the Reynolds number applied, at an intermediate level in the bulk $\left(\Delta y^{+} \approx 5.8\right)$. In Fig. 13 the corresponding Reynolds stresses $\overline{u^{\prime} u^{\prime}}$ and $\overline{u^{\prime} v^{\prime}}$ for this setup are shown. 


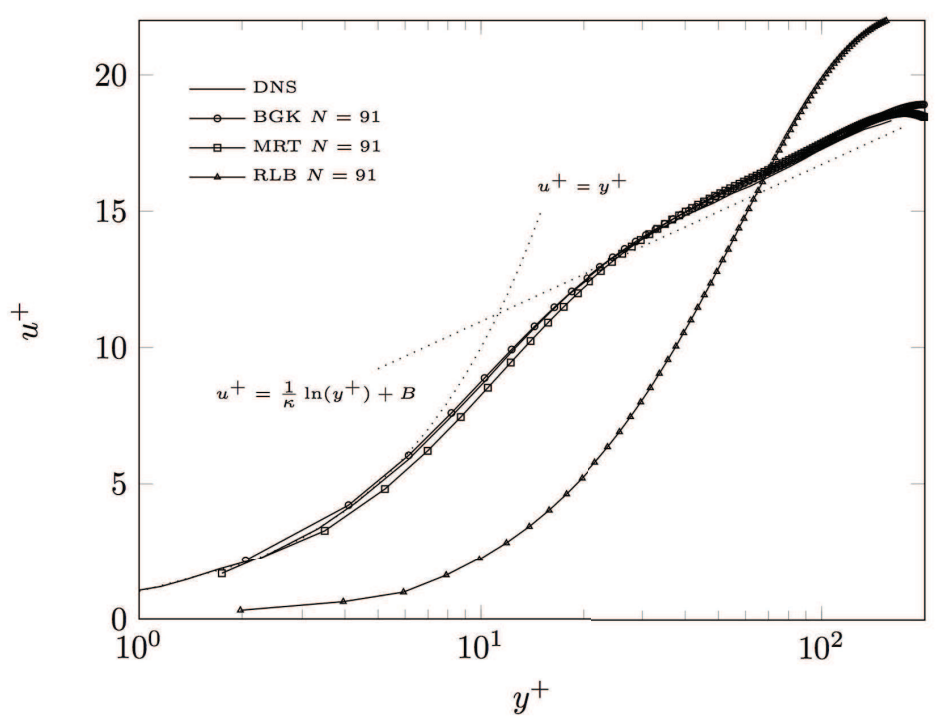

Figure 14: Mean velocity profiles of turbulent channel flow at $R e_{\tau}=180$ and $N=91$ compared to the DNS of Kim et al. [22].

The $\overline{u^{\prime} u^{\prime}}$ stresses are underestimated by both schemes, which is caused by the low resolution applied. The BGK model has a slightly better agreement with the reference data compared to the MRT model over the whole channel height. Although both LBM models predict reduced magnitudes for $\overline{u^{\prime} u^{\prime}}$ compared to the reference data, the wall normal location of the peak value of this stress component was found correctly. The mean $\overline{u^{\prime} v^{\prime}}$ stresses are predicted with good accuracy by the BGK and MRT scheme when compared to the reference DNS.

For a resolved simulation at $R e_{\tau}=180$ the resolution was increased to $N=91$, which corresponds to the setup of Bespalko [2] and gives a resolution of $\Delta y^{+} \approx 2$ within the bulk and wall region. The simulations with the MRT and the BGK model run stable. For the mean velocity profiles very good agreement of the BGK and MRT results with reference DNS can be found. While the MRT model slightly underpredicts the velocities close to the wall, an over estimation of the mean velocities is found in the bulk flow. On the other hand the BGK scheme overpredicts the velocity field slightly in the wall-nearest region whilst excellent agreement was found in the bulk region when compared to the DNS data, see Fig. 14.

Fig. 15 shows that the $\overline{u^{\prime} u^{\prime}}$ and $\overline{u^{\prime} v^{\prime}}$ stresses predicted by the BGK scheme are in good agreement with the reference DNS data. Differences in the amplitude of the stresses in the bulk region can be related to the higher predicted $R e_{\tau, \text { real }}$, see Table 2 . For the MRT scheme, the peak value and the magnitude of the $\overline{u^{\prime} u^{\prime}}$ stress are in very good agreement with the reference data. For the $\overline{u^{\prime} v^{\prime}}$ stress minor deviations from the reference DNS are visible for both the MRT and BGK scheme. Since this resolution is nearly the same as 


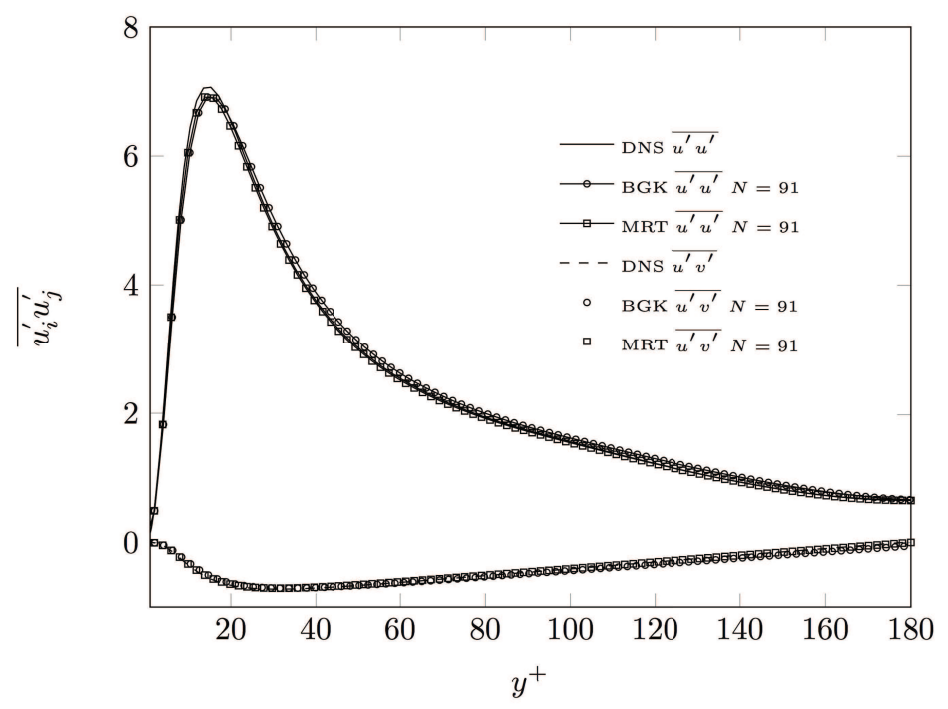

Figure 15: Mean Reynolds stresses for turbulent channel flow at $R e_{\tau}=180$ and $N=91$ compared to the DNS of Kim et al. [22].

in the numerical setup applied by Wang et al. [44], who found excellent agreement for the Reynolds stresses by applying the MRT scheme at $R e_{\tau}=180$, we assume that for this low Reynolds number both the different forcing approach as well as the straight velocity boundary condition have a negative influence on the accurate prediction of the stresses in vicinity of the wall in our case.

By increasing the friction Reynolds number up to $R e_{\tau}=395$ with $N=91$, again the BGK model shows good agreement with the reference data of Moser et al. [35] for both the velocity field and the Reynolds stresses, see Figs. 16 and 17. Discrepancy of the peaks predicted by the BGK scheme are $\approx 7.9 \%$ and $\approx 11.2 \%$ for the $\overline{u^{\prime} u^{\prime}}$ and $\overline{u^{\prime} v^{\prime}}$ stresses respectively. The MRT model underpredicts the mean flow field in vicinity of the wall $y^{+}<40$, while the bulk region was overpredicted. Regarding the Reynolds stresses, see Fig. 17, one can see that the $\overline{u^{\prime} u^{\prime}}$ as well as the $\overline{u^{\prime} v^{\prime}}$ stresses are grossly and irregularly overpredicted by the MRT scheme for the same amount of physical time averages. For $R e_{\tau}=395$ and $N=91$ the MRT model converged to a state exhibiting unphysical oscillations. A similar behavior was found when increasing the resolution to $N=151$, see Figs. 18 and 19. The difference of the $\overline{u^{\prime} u^{\prime}}$ and $\overline{u^{\prime} v^{\prime}}$ stresses predicted by the BGK scheme are only $\approx 6.8 \%$ and $\approx 9.1 \%$ respectively compared to the reference DNS data. For the MRT scheme, the mean velocity profile is under predicted in the wall-nearest region, while the mean velocity exceeds the DNS data in the bulk region by $\approx 6 \%$. The stresses are similar as for $N=91$, yet even for the same physical time of averaging, more fluctuations are observed.

In Fig. 20 the velocity field predicted by the BGK scheme for $R e_{\tau}=590$ and $N=91$ is illustrated. Although the resolution is quite coarse at this Reynolds number $\left(y^{+} \approx 6.48\right)$, the simulation covers the fully turbulent state, see also Fig. 8 . The agreement of the 


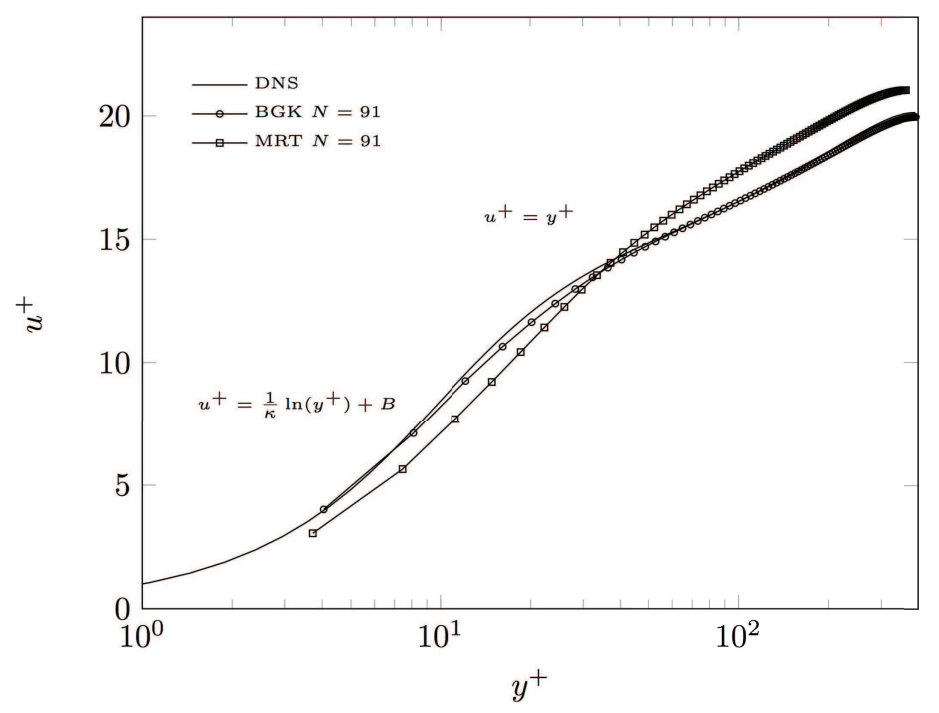

Figure 16: Mean velocity profiles of turbulent channel flow at $R e_{\tau}=395$ and $N=91$ compared to the DNS of Moser et al. [35].

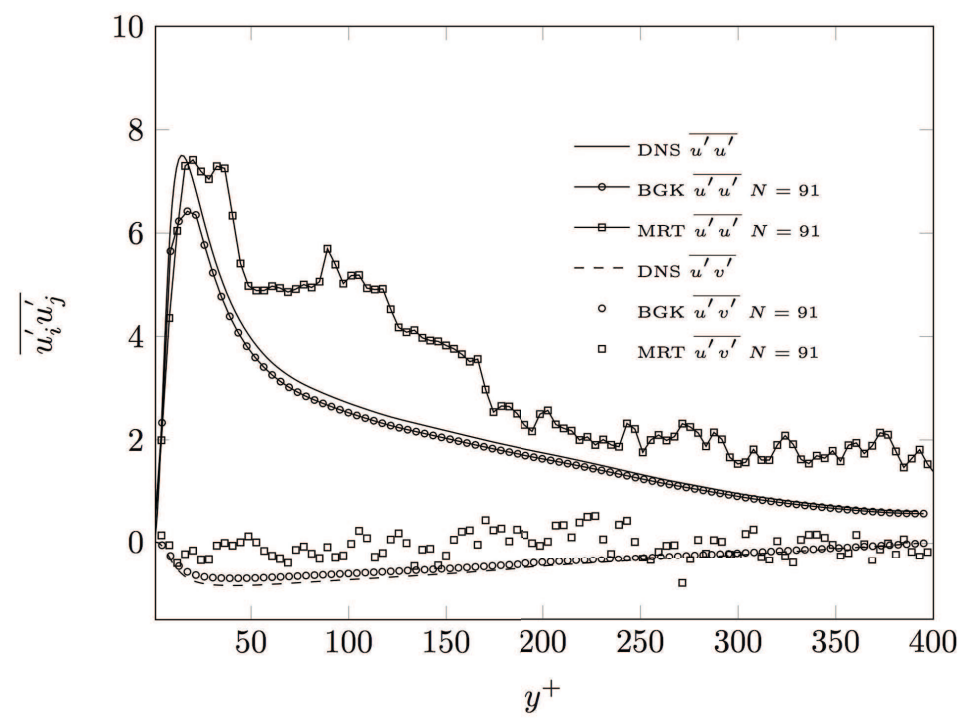

Figure 17: Mean Reynolds stresses of turbulent channel flow at $R e_{\tau}=395$ and $N=91$ compared to the DNS of Moser et al. [35].

mean velocity field with the reference DNS data is found to be excellent, see Fig. 20, while the stresses are under-predicted by almost $20 \%$ over the whole channel height, see Fig. 21. Beyond that, the location of the stress maximum is shifted for the $\overline{u^{\prime} u^{\prime}}$ and $\overline{u^{\prime} v^{\prime}}$ stresses, which is most probably caused by the very low resolution of $\Delta y^{+} \approx 6.48$. The 


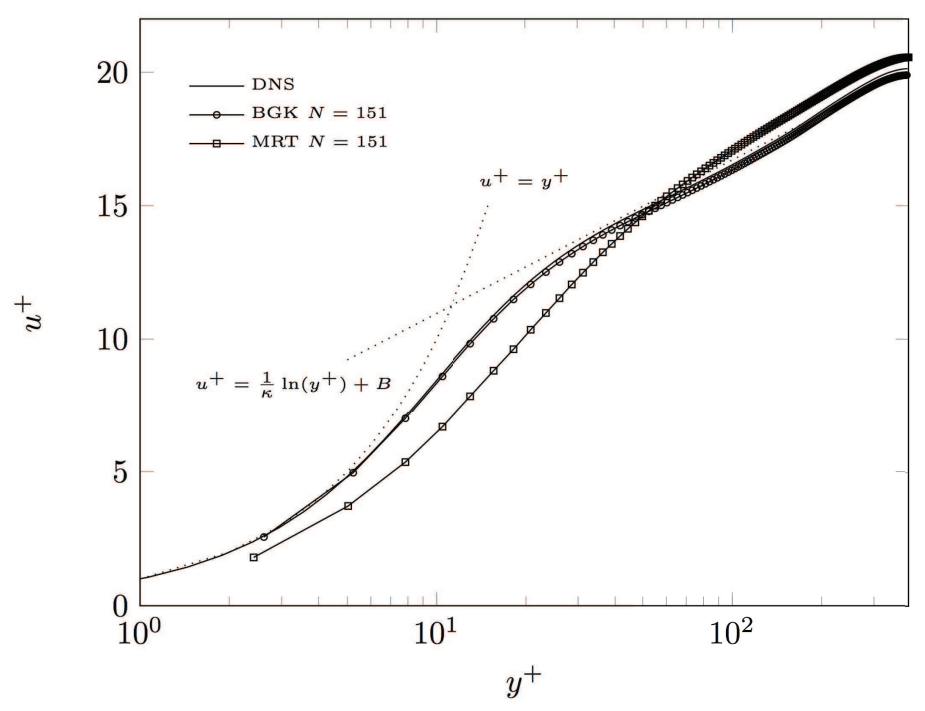

Figure 18: Mean velocity profiles of turbulent channel flow at $R e_{\tau}=395$ and $N=151$ compared to the DNS of Moser et al. [35].

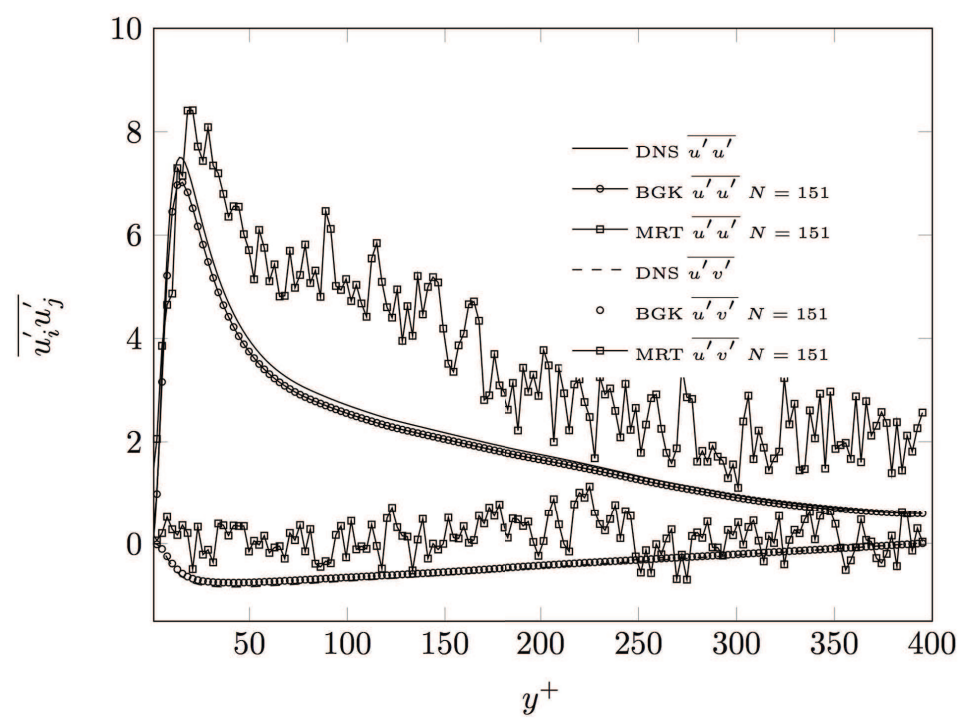

Figure 19: Mean Reynolds stresses of turbulent channel flow at $R e_{\tau}=395$ and $N=151$ compared to the DNS of Moser et al. [35].

MRT model assumed an unphysical state for the resolution $N=91$, which is evident from unphysical spurious oscillations, that propagate throughout the whole channel, see also Figs. 9 and 21. For this setup, even the general quality of the evolution of the stresses is not in accordance with the DNS data. The quality of the results does not change by 


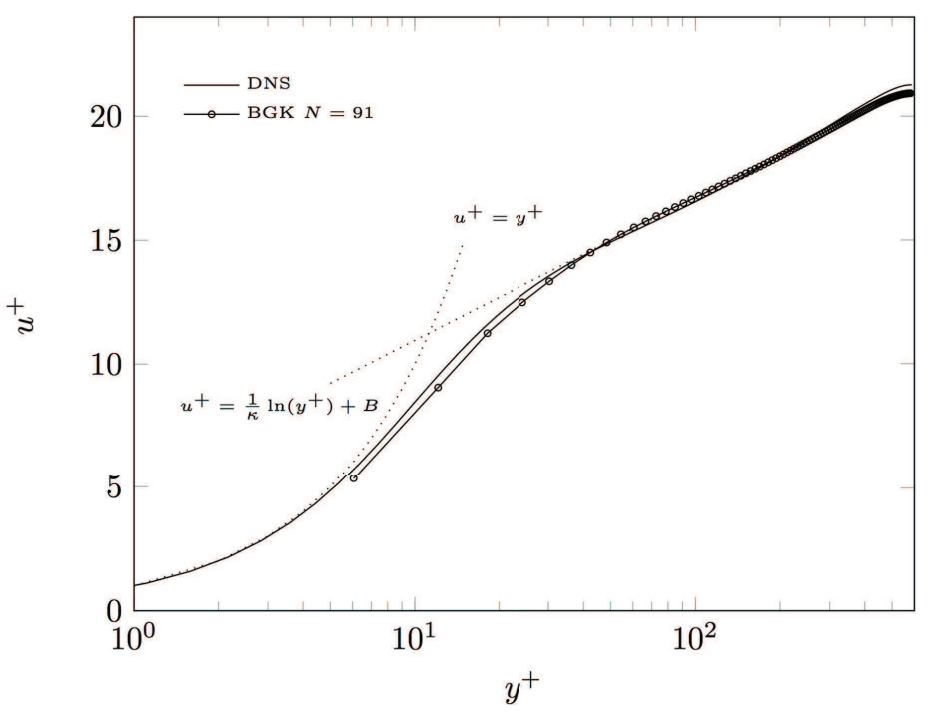

Figure 20: Mean velocity profiles of turbulent channel flow at $R e_{\tau}=590$ and $N=91$ compared to the DNS of Moser et al. [35].

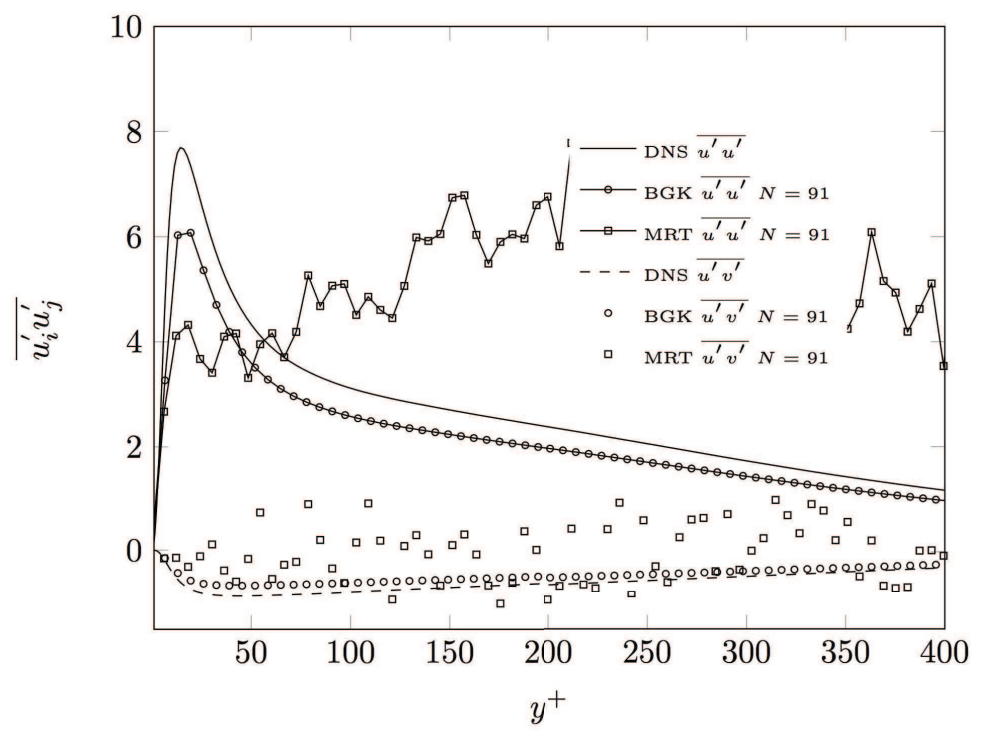

Figure 21: Mean Reynolds stresses of turbulent channel flow at $R e_{\tau}=590$ and $N=91$ compared to the DNS of Moser et al. [35].

increasing the resolution to $N=151$, see Figs. 22 and 23, except that the uncertainty of the stresses predicted by the BGK scheme decreased to $\approx 10 \%$.

Recently, Gehrke et al. [12] presented similar results when computing the turbulent channel flow. Although the boundary conditions were not identical, the MRT model 


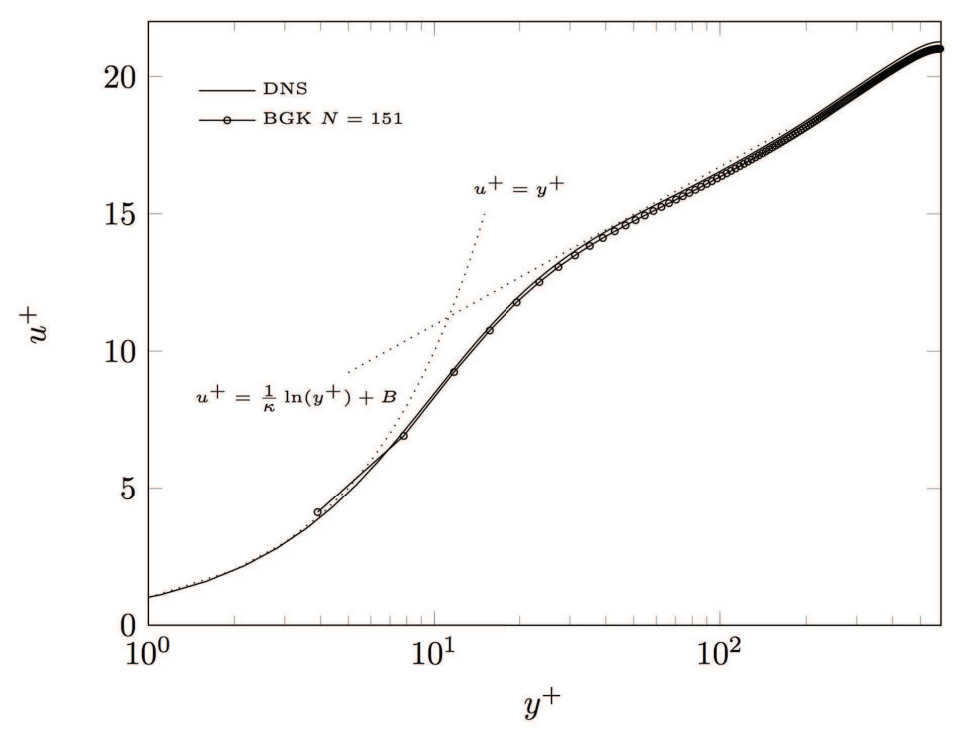

Figure 22: Mean velocity profiles of turbulent channel flow at $R e_{\tau}=590$ and $N=151$ compared to the DNS of Moser et al. [35].

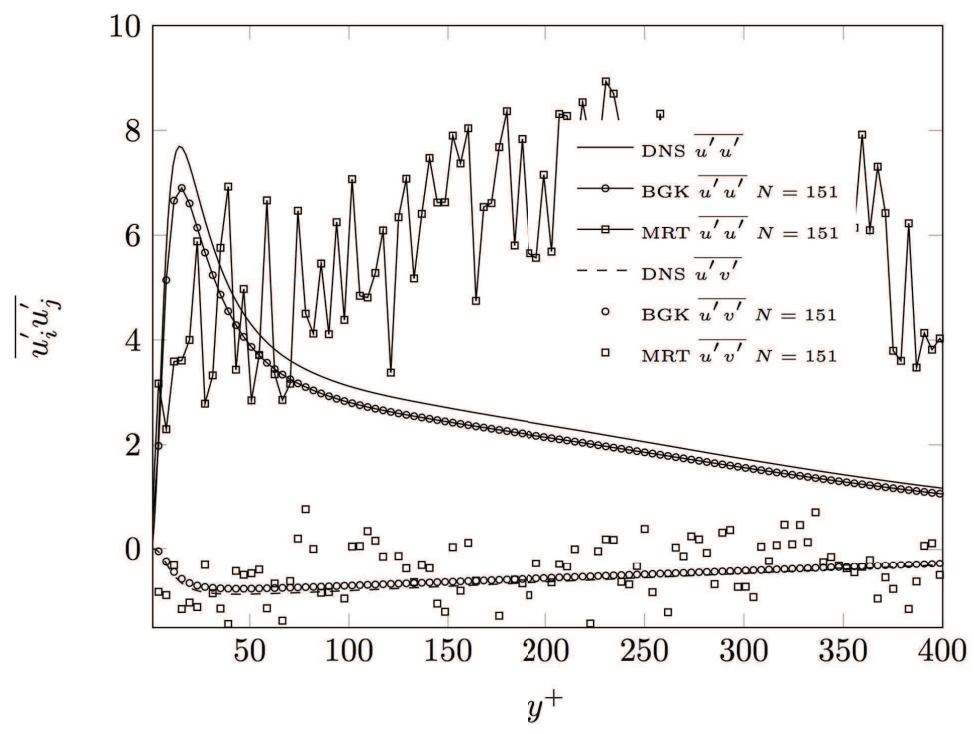

Figure 23: Mean Reynolds stresses of turbulent channel flow at $R e_{\tau}=590$ and $N=151$ compared to the DNS of Moser et al. [35].

produced similar spurious noise in their simulations and the BGK model was able to reproduce the reference data with increased accuracy. The independency of the boundary conditions indicate, that the problems of the MRT model are intrinsic when calculating turbulent flows directly on different mesh levels. 


\subsection{Spectral analysis}

We analyze the wall-normal energy distribution in spectral space. Fig. 24 shows the normalized energy for the BGK and MRT scheme at all investigated Reynolds numbers and $N=91$. Similarly to the energy spectra depicted in Section 3, the MRT model shows an unphysical increase of energy in the high-frequency range for $R e_{\tau}=395$ and $R e_{\tau}=590$. Since the energy is normalized, it is visible, that a high amount of energy is shifted from the low wavenumber range towards the high wavenumber range for $R e_{\tau}=395$, which is even more apparent for $R e_{\tau}=590$. These high wavenumber fluctuations of the velocity represent the unphysical flow state shown in Fig. 9.

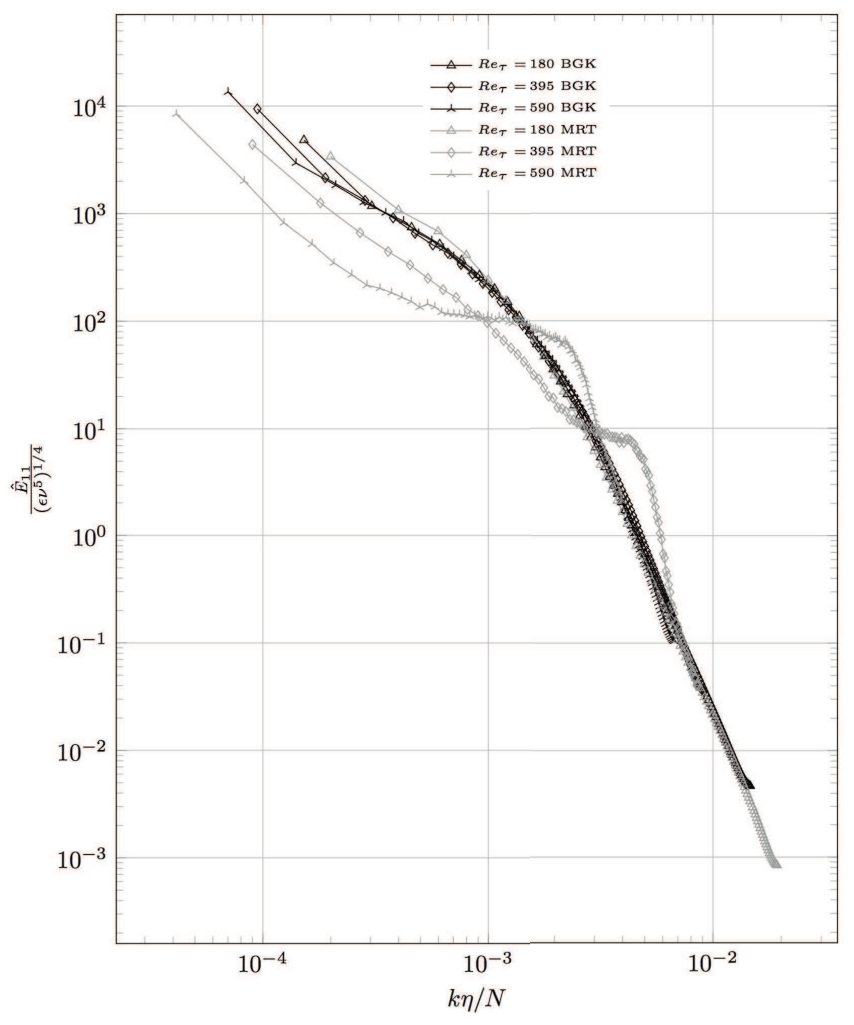

Figure 24: Wall normal spectra of the normalized energy of the BGK and MRT scheme for all investigated Reynolds numbers and $N=91$.

\section{Conclusions}

In this paper, the accuracy and stability of three popular lattice schemes, the BGK, MRT and RLB approach, when employed for simulations of turbulent flows were investigated. Homogeneous isotropic turbulence was considered by computing the Taylor-Green vor- 
tex. Different resolutions and Reynolds numbers were considered. As it was found earlier by several authors, the BGK scheme [7] suffers from numerical instabilities for increasing Reynolds numbers at fixed resolutions. The reasons are unphysical moments, which are amplified exponentially in time for under-resolved setups. Nevertheless, when increasing the resolution, mesh convergence and accurate results were obtained. Due to the low dissipation found for the BGK model at medium and well resolved setups, the adaption of advanced turbulence models, such as ADM, seems to be promising for this type of collision operator. To circumvent the instabilities of the BGK scheme at low resolutions, the MRT scheme [8] was derived. In our investigation this expected feature of the MRT scheme was confirmed, but only in under-resolved test cases. Increasing the resolution towards DNS lead to unstable simulations, and no mesh convergence could be achieved with the MRT-LBM for of homogeneous turbulence considered in this work. The RLB scheme allowed for stable simulations at all resolutions and Reynolds numbers considered for the Taylor-Green vortex and mesh convergence was achieved. The RLB suffers from large numerical dissipation, which grossly decreases accuracy.

By computing turbulent channel flows at $R e_{\tau}=180, R e_{\tau}=395$ and $R e_{\tau}=590$ for varying resolutions, some features found for the Taylor-Green vortex could be confirmed. For the rather low Reynolds number of $R e_{\tau}=180$, very good agreement was found for both the BGK and MRT scheme, while the MRT scheme showed even better agreement with the reference data. By increasing the Reynolds number to $R e_{\tau}=395$ and $R e_{\tau}=590$, no mesh convergence for the MRT model was found. While the BGK model lead to reasonable and good results, the MRT model showed the appearance of spurious velocity oscillations in the bulk. For an increasing Reynolds number these oscillations were also found in the vicinity of the wall. Although the MRT model did not became unstable, the simulations reproduced an unphysical state. The RLB model was only applied for the lowest Reynolds number at $R e_{\tau}=180$ and it was found, that the flow field resulted in a laminar state, which was confirmed by the velocity profile and the temporal evolution of the $R e_{\tau}$. Additional work has to be performed on the validation of the forcing approach to reach the target turbulence state. Since the mean velocity profile is maybe affected by the development of spurious oscillations, mode coupling between such spurious fluctuations and forcing oscillations may occur. Different initialization techniques and Reynolds numbers should be applied by computing the turbulent channel flow with the RLB scheme to test the general application of this model for wall-bounded turbulent flows.

For the MRT model it is believed, that the high order moments are responsible for the absence of mesh convergence at high Reynolds numbers employing a resolved numerical setup with diffusive scaling, see Sections 3 and 4. It was shown, that the MRT model produces an unphysical amount of energy in the high frequency space. Due to the developing strong oscillations, the classical energy transfer of a turbulent flow is disturbed. The results obtained in this work can be useful for further development of turbulence models and underline the suitability of the BGK scheme as an efficient and accurate DNS tool for turbulent fluid flows. 


\section{References}

[1] C. K. Aidun and J. R. Clausen. Lattice-boltzmann method for complex flows. Annual Review of Fluid Mechanics, 42:439-472, 2010.

[2] D. J. Bespalko. Validation of the Lattice Boltzmann Method for Direct Numerical Simulation of Wall-Bounded Turbulent Flows. dissertation, Queen's University, 2011.

[3] P. L. Bhatnagar, E. P. Gross, and M. Krook. A model for collision processes in gases. i. small amplitude processes in charged and neutral one-component systems. Phys. Rev., 94:511-525, 1954.

[4] M. E. Brachet. Direct simulation of the three-dimensional turbulence in th taylor-green vortex. Fluid Dynamics Research, 8:1-8, 1991.

[5] O. Cabrit. Direct simulations for wall modeling of multicomponent reacting compressible turbulent flows. Phys. Fluids, 21:055108, 2009.

[6] A. Caiazzo. Analysis of lattice boltzmann initialization routines. J. Stat. Ph., 121:37-48, 2005.

[7] S. Chen and G. D. Doolen. Lattice boltzmann method for fluid flows. Annu. Rev. Fluid Mech., 30:329-364, 1998.

[8] D. D’Humiéres, I. Ginzburg, M. Krafczyk, P. Lallemand, and L.-S. Luo. Multiple-relaxationtime lattice boltzmann models in three dimensions. Phil. Trans. R. Soc. Lond. A, 361:437-451, 2002.

[9] B. Dorschner, F. Bösch, S. S. Chikatamarla, K Boulouchos, and I. V. Karlin. Entropic multirelaxation time lattice boltzmann model for complex flows. J. Fluid Mech., 801:623-651, 2016.

[10] B. Dorschner, N. Frapolli, S. S. Chikatamarla, and I. V. Karlin. Grid refinement for entropic lattice boltzmann models. Phys. Rev. E, 94:053311, 2016.

[11] R. K. Freitas, A. Henze, M. Meinke, and W. Schröder. Anaysis of lattice-boltzmann methods for internal flows. Comp. Fl., 47:115-121, 2011.

[12] M. Gehrke, C.F. Janßen, and T Rung. Scrutinizing lattice boltzmann methods for direct numerical simulations of turbulent channel flows. Computers $\mathcal{E}$ Fluids, 156:247-263, 2017.

[13] M. Geier, M. Schoenherr, A. Pasquali, and M. Krafczyk. The cumulant lattice Boltzmann equation in three dimensions: theory and validation. Computers and Mathematics with Applications, 70:507, 2015.

[14] M. C. Geier. Ab initio derivation of the cascaded lattice Boltzmann automaton. dissertation, Universität Freiburg, 2006.

[15] Z. Guo, S. Baochang, and W. Nengchao. Lattice bgk model for incompressible navier stokes equation. J. Comp. Ph., 165:288-306, 2000.

[16] Z. Guo and S. Shu. Lattice Boltzmann Method and its Applications in Engineering. World Scientific, 2013.

[17] D. Hänel. Molekulare Gasdynamik. Springer-Verlag, 2004.

[18] X. He and L.-S. Luo. Lattice boltzmann model for the incompressible navier stokes equation. J. Stat. Phys., 88:927-944, 1997.

[19] X. He and G. D. Shan, X.and Doolen. Discrete boltzmann equation model for nonideal gases. Phys. Rev. E, 57:R13-R16, 1998.

[20] T. Henn, G. Thäter, W. Dörfler, H. Nirschl, and M.J. Krause. Parallel dilute particulate flow simulations in the human nasal cavity. Computers $\mathcal{E}$ Fluids, 124:197-207, 2016.

[21] I. V. Karlin, F. Bösch, and S. S. Chikatamarla. Gibbs' principle for the lattice-kinetic theory of fluid dynamics. Phys. Rev. E, 90:031302, 2014.

[22] J. Kim, P. Moin, and R. Moser. Turbulence statistics in fully-developed channel flow at low reynolds-number. J. Fluid Mech., 177:133-166, 1987. 
[23] M.J. Krause, T. Gengenbach, and V. Heuveline. Hybrid Parallel Simulations of Fluid Flows in Complex Geometries: Application to the Human Lungs, volume 6586. Springer Berlin / Heidelberg, 2011.

[24] M.J. Krause and V. Heuveline. Parallel Fluid Flow Control and Optimisation with Lattice Boltzmann Methods and Automatic Differentiation. Computers and Fluids, 80(0):28-36, 2013.

[25] A. J. C. Ladd and R. Verberg. Lattice-boltzmann simulations of particle-fluid suspensions. J. Stat. Phys., 104:1191-1251, 2001.

[26] P. Lallemand and L. S. Luo. Theory of the lattice boltzmann method: Dispersion, dissipation, isotropy, galilean invariance, and stability. Phys. Rev. E, 61:6546, 2000.

[27] P. Lammers, K. N. Bernonov, R. Volkert, G. Brenner, and F. Durst. Lattice bgk direct numerical simulation of fully developed turbulence in incompressible plane channel flow. Comp. Fl., 35:1137-1153, 2006.

[28] J. Latt. Hydrodynamic Limit of Lattice Boltzmann Equations. dissertation, Université de Genève, 2007.

[29] J. Latt and B. Chopard. Lattice boltzmann method with regularized non-equilirbium distribution functions. Mathematics and Computer in Simulation, 72:165-168, 2006.

[30] J Latt, B. Chopard, O. Malaspinas, M. Deville, and A. Michler. Straight velocity boundaries in the lattice boltzmann method. Phys. Rev. E, 77:056703, 2008.

[31] O. Malaspinas and P. Sagaut. Advanced large-eddy simulation for lattice boltzmann methods: The approximate deconvolution model. Phys. Fluids, 23:105103, 2011.

[32] O. Malaspinas and P. Sagaut. Consistent subgrid scal modelling for lattice boltzmann methods. J. Fluid Mech., 700:514-542, 2012.

[33] O. Malaspinas and P. Sagaut. Wall model for large-eddy simulation based on the lattice boltzmann methodl. J. Comp. Phys., 275:25-40, 2014.

[34] M. J. Mathias. Fluid Flow Simulation and Optimisation with Lattice Boltzmann Methods on High Performance Computers. dissertation, Universität Karlsruhe, 2010.

[35] R. D. Moser, J. Kim, and N. N. Mansour. Direct numerical simulation of turbulent channel flow up to $r e_{\tau}=590$. Phys. Fl., 11:943-945, 1999.

[36] S. B. Pope. Turbulent Flows. Cambridge University Press, 2013.

[37] D. Ricot, V. Maillar, and C. Bailly. Numerical simulation of unsteady cavity flow using lattice boltzmann method. In 8th AIAA/CEAS Aeroacoustics Conference \& Exhibit, 2002.

[38] D. Ricot, S. Marie, P. Sagaut, and C. Bailly. Lattice boltzmann method with selective viscosity filter. J. Comp. Phys, 228:4478-4490, 2009.

[39] P. Sagaut. Toward advanced subgrid models for lattice boltzmann based large-eddy simulation: Theoretical formulations. Computers and Mathematics with Applications, 59:2194-2199, 2010.

[40] S. Stolz and N. A. Adams. An approximate deconvolution procedure for large-eddy simulation. Phys. Fluids, 11:1699, 1999.

[41] M. C. Sukop and D. T. Thorne. Lattice Boltzmann Modeling. Springer-Verlag, 2006.

[42] Takahiro Tsukahara, Yohji Seki, Hiroshi Kawamura, and Daisuke Tochio. Dns of turbulent channel flow at very low reynolds numbers. In TSFP DIGITAL LIBRARY ONLINE. Begel House Inc., 2005.

[43] D. A. Waldrow. Lattice-Gas Cellular Automata and Lattice Boltzmann Models: An Introduction. Springer-Verlag, 2000.

[44] L. P. Wang, C. Peng, Z. Guo, and Z. Yu. Lattice boltzmann simulation of particle-laden turbulent channel flow. Comp. Fl., 124:226-236, 2016. 


\section{Repository KITopen}

Dies ist ein Postprint/begutachtetes Manuskript.

Empfohlene Zitierung:

Nathen, P.; Gaudlitz, D.; Krause, M. J.; Adams, N. A.

On the Stability and Accuracy of the BGK, MRT and RLB Boltzmann Schemes for the Simulation of Turbulent Flows.

2018. Communications in computational physics, 23.

doi: $10.5445 / / R / 1000083814$

Zitierung der Originalveröffentlichung:

Nathen, P.; Gaudlitz, D.; Krause, M. J.; Adams, N. A.

On the Stability and Accuracy of the BGK, MRT and RLB Boltzmann Schemes for the Simulation of Turbulent Flows.

2018. Communications in computational physics, 23 (2), 846-876.

doi:10.4208/cicp.OA-2016-0229 